\title{
Yield and Response of Bean Breeding Lines for Drought Tolerance to Field Diseases
}

\author{
Winnyfred Amongi ${ }^{1}$, Clare Mukankusi $^{1}$, Sulaiman Sebuliba ${ }^{1}$, Brenda Nakyanzi $^{1}$, Claire Naluwooza ${ }^{1}$ \\ \& Gerald Baguma ${ }^{2}$ \\ ${ }^{1}$ International Center for Tropical Agriculture, Kampala, Uganda \\ ${ }^{2}$ Kachwekano Zonal Research and Development institute, Kabale, Uganda \\ Correspondence: Winnyfred Amongi, International Center for Tropical Agriculture (CIAT), P.O. Box 6247, \\ Kampala, Uganda. Tel: 256-774-633-411. E-mail: w.amongi@cgiar.org
}

Received: February 27, 2019 Accepted: June 14, 2019 Online Published: August 15, 2019

doi:10.5539/jas.v11n13p81 URL: https://doi.org/10.5539/jas.v11n13p81

\begin{abstract}
Climate change has resulted in an increase in the intensity of droughts and rains, and higher temperatures which are adversely affecting crop production in Africa. It has also influenced the distribution and increased the occurrence of disease and pest epidemics. Common bean (Phaseolus vulgaris L.) is especially sensitive to these changes. Most released varieties are not well adapted to environmental extremes and extended periods of drought in particular has become a major constraint. In this study, 462 advanced breeding lines developed for drought tolerance were evaluated for yield, agronomic traits and disease response in two contrasting agro-ecologies, Kawanda in the Lake Victoria crescent and Kachwekano in the southwestern highlands. Analysis of variance indicated significant differences $(\mathrm{P}<0.05)$ among lines for most variables and environments $(\mathrm{P}<$ 0.001). Performance was better at Kachwekano than Kawanda with mean yields ranging from 928 to $2251 \mathrm{~kg}$ $\mathrm{ha}^{-1}$, and 698 to $2036 \mathrm{~kg} \mathrm{ha}^{-1}$ respectively. Angular leafspot, common bacterial blight and rust diseases varied between locations, and among lines with no visible to sever symptoms. Based on Wricke's ecovalence estimates for stability, SCN20, SCN13, SEN114, SEC40 and SEC35 expressed yield stability and superiority. Of the 462 lines, $6.3 \%$ maintained $>1500 \mathrm{~kg} \mathrm{ha}^{-1}$, the minimum anticipated commercial yield of new varieties, in all the trials, both seasons and in each year; $5.0 \%$ also maintained above average yields. The most outstanding (1629-3944 $\mathrm{kg} \mathrm{ha}^{-1}$ ) were; DAB299, DAB291, DAB234, DAD34, DAB478, DAB487, DAB543, DAB231, SCN20, SCR66, SCR60, and SER335. These are recommended for development of new varieties.
\end{abstract}

Keywords: diseases, drought, Phaseolus vulgaris, yield stability

\section{Introduction}

Common bean (Phaseolus vulgaris L.) is a staple crop that feeds more than 100 million people in Africa (Buruchara et al., 2011) but biotic and abiotic stresses are causing significant yield reductions across the production area (Broughton et al., 2003). Of the abiotic stresses, drought has progressively become one of the major constraints. Unless mitigation strategies are adopted, further declines in yield are anticipated because drought has become more frequent and prolonged (National Adaptation Programmes of Action [NAPA], 2007; East African Community [EAC], 2011). One strategy is irrigation but it is not an option that most farmers can afford. Breeding drought-tolerant varieties is therefore considered a better and more sustainable alternative. During the two main annual growing seasons, this study examined the performance of 462 lines bred for drought tolerance.

Besides losses to drought and in spite of crop improvement programs, yields of bean in farmers' fields in East and Central Africa, 500 to $900 \mathrm{~kg} \mathrm{ha}^{-1}$, are well below the potential obtainable from new varieties, $1.5-2.0 \mathrm{t} \mathrm{ha}^{-1}$ (International Center for Tropical Agriculture [CIAT], 2008). In experimental systems, maximum yields of 4.0 and $6.0 \mathrm{t} \mathrm{ha}^{-1}$ have been demonstrated for bush and climbing types of beans respectively (Beebe, Rao, Blair, \& Acosta-Gallegos, 2013). Low farmer yield is attributed to poor agronomic practices, and abiotic as well as biotic plant stress. Drought was affecting 40\% of the bean growing areas in Africa by 2003 (Broughton et al., 2003). The affected area has since increased (United Nation Framework Convention on Climate Change [UNFCCC], 2007; EAC, 2011) and yield reductions $>80 \%$ have been reported (Singh, 2007; Amongi, Nkalubo, Ochwo-Ssemakula, Gibson, \& Edema, 2014; Asfaw \& Blair, 2014). Kiwuka, Bukenya-Ziraba, Namaganda, and 
Wasswa (2012) reported that drought was starting to affect the adaptation of common bean to Ugandan environments. Changing climates are also altering the occurrence of certain biotic constraints (Garrett, Dendy, Frank, Rouse, \& Travers, 2006; Gautam, Bhardwaj, \& Rohitashw, 2013; Paparu et al., 2014), so multiple stress tolerant lines are now sought for lessening the effect of these diverse challenges. The common sources of biotic stress are bean fly (Ophiomyia phaseoli), aphids (Aphis fabae), weevils (Acanthoscelides obtectus), fungal, bacterial and viral diseases including anthracnose (Colletotrichum lindemuthianum), angular leaf spot (ALS) (Phaeoisariopsis griseola), common bacterial blight (CBB) (Xanthomonas axonopodis pv. phaseoli), bean common mosaic virus and its necrotic strain, and root rots (Fusarium solani, Pythium ultimum and Rhizoctonia solani, Sclerotium species). ALS, rot rots, and anthracnose may cause $50 \%$ to $100 \%$ yield loss (Uganda Export Promotion Board [UEPB], 2005; Opio, Ugen, Kyamanywa, David, \& Mugisa-Mutetikka, 2001; Nkalubo, Melis, Laing, \& Opio, 2007; Mukankusi, 2008; Paparu et al., 2014).

Drought tolerance is a highly specific trait where genotypes tend not to be adapted to a broad range of environmental conditions (Acquaah, 2007). In beans, drought tolerance appears to result from a deeper root system, more sensitive stomatal control and a capacity to remobilize stored carbohydrates under stress (Acquaah, 2007; Beebe et al., 2013). The crop has a wide genetic base (Beebe et al., 2013; Kiwuka et al., 2012) with genetic differences in seed weight, leaf proline content, stay-green, root spread and depth, all of which are associated with drought tolerance (Thomas, 1983; Badr, 2005). However, the effectiveness with which carbohydrates are translocated and partitioned as a preferred selection method has been emphasized by Beebe et al. (2013), Asfaw and Blair (2014), and Amongi et al. (2014). This entails the comparison of the performance of breeding lines under stress and non-stress conditions using seed and biomass yield measurements (White \& Singh, 1991; Beebe et al., 2013).

Breeding programs in East and Central Africa have only recently initiated the screening of existing germplasm for crop improvement of common bean (Gathu, Karuri, \& Njage, 2012; Amongi et al., 2014; Asfaw \& Blair, 2014). By contrast, in South America, breeding for drought tolerance in beans has over a 20 -year history and through the program at the International Center for Tropical Agriculture (CIAT) in Colombia (Beebe, Rao, Cajiao, \& Grajales, 2008), breeding and selection have resulted in many drought tolerant lines (Blair et al., 2012; Beebe et al., 2013) belonging to several market classes. To support similar activities in Africa, CIAT-Uganda, under the umbrella of the Pan Africa Bean Research Alliance (PABRA) (Buruchara et al., 2011; www.pabra.ciatafrica.org), has obtained drought-tolerant advanced lines from South America to identify superior genotypes (lines) for distribution in the PABRA network (Buruchara et al., 2011). In this study, genotypes improved for drought tolerance were phenotyped for yield, agronomic traits and tolerance to prevailing field diseases.

\section{Material Studied}

Advanced bush bean breeding lines of $P$. vulgaris developed for drought tolerance by the CIAT bean breeding program in South America were grouped as; (i) Drought-tolerant Andean beans (DAB) Set2 with 185 lines; (ii) DAB Set4 with 95 lines; (iii) Black beans with 33 lines consisting of black grain with bean common mosaic virus (BCMV) recessive gene (NCB), black drought-tolerant beans with BCMV resistant gene (SCN) and black drought-tolerant beans (SEN); (iv) Colored beans with 15 lines consisting of lines resistant to BCMV (SIN) and different colored drought-tolerant beans (SEC); (v) Assorted with 55 lines consisting of Drought-adapted Andean beans for Africa (DAA), Drought tolerant Andean (DAD), large seed (GRR) and large seed crosses block (GGB); and (vi) Small red beans with 79 lines consisting of drought-tolerant small red bush beans with BCMV recessive gene (SCR), drought-tolerant red small bush beans (SER), and drought and physiology beans (SEF). A popular commercially grown variety, CAL96, was included as a local yield check.

\section{Area Descriptions}

The study was conducted in two locations (L) representing contrasting agro-ecologies in Uganda. At the CIAT station based at the National Agricultural Research Laboratories (NARL), Kawanda, located at $0^{\circ} 25^{\prime} \mathrm{N}, 32^{\circ} 31^{\prime} \mathrm{E}$ and at an elevation of $1190 \mathrm{~m}$ above sea level (asl), and at Kachwekano Zonal Agricultural Research and Development Institute (KAZARDI), Kabale, located at $1^{\circ} 15^{\prime} \mathrm{S}, 29^{\circ} 57^{\prime} \mathrm{E}$ at an elevation of $2200 \mathrm{~m}$ asl. Weather data was collected on-station. The total amount of rainfall received in the 2015 September-December season was $792.3 \mathrm{~mm}$ at Kawanda. In the 2016 April to July season, $602.3 \mathrm{~mm}$ and $179.8 \mathrm{~mm}$ of rainfall were received at Kawanda and Kachwekano respectively. In the two seasons at Kawanda, mean minimum and maximum temperatures were 19.2 and $27.6^{\circ} \mathrm{C}$, and 18.1 and $28.2^{\circ} \mathrm{C}$, respectively. At Kachwekano, mean temperatures were $13.7{ }^{\circ} \mathrm{C}$ and $22.1{ }^{\circ} \mathrm{C}$. Weather data for the trials established in 2012 at Kawanda and in the September-December season in 2015 at Kachwekano are not available. 


\section{Methods and/or Techniques}

\subsection{Trial Establishment}

Trials were established during the first (A) and second (B) rainy seasons, April to July and September to December, respectively. DAB Set2 was the only group planted at Kawanda in 2012A and B. All the other trials were established in 2015B and 2016A at Kachwekano and Kawanda. The lines were laid out in an alpha lattice design with two replications. Plots representing each line within a replication were of 3 rows by $3 \mathrm{~m}$ in length; row and plant spacing were $50 \mathrm{~cm}$ and $10 \mathrm{~cm}$ respectively. Each trial was weeded thrice and an insecticide, Dimethoate and two fungicides (Mancozeb and Ridomil) were applied weekly until flowering. The recommended manufacturer's rate was used for each pesticide. Granular N:P:K 17:17:17 fertilizer was hand applied just before planting at the rate of $125 \mathrm{~kg} \mathrm{ha}^{-1}$.

\subsection{Data Collection}

Data for yield, disease and growth variables were collected at specific intervals based on the bean trait dictionary (Integrated Breeding Platform [IBP], 2013). Days to flowering and physiological maturity were recorded as number of days from planting to the day when $50 \%$ of plants had at least one flower and number of days from planting to the day when the first pods began to discolor in $50 \%$ of the plants, respectively (CIAT, 1987; IBP, 2013). Growth vigor was recorded on 1-9 scale where $1=$ Excellent, $3=$ Good, $5=$ Intermediate, $7=$ Poor, $9=$ Very poor (CIAT 1987). Seed collection for yield began when $90 \%$ of the pods had changed color to yellow (Munoz-Perea et al., 2006). The seeds were sun dried to $13 \%$ moisture content and sorted before recording clean seed weight per plot $(\mathrm{g})$.

The response of plants to field diseases; angular leaf spot (ALS), common bacterial blight (CBB) and rust, was assessed using a CIAT disease evaluation scale of 1-9 (CIAT, 1987; IBP, 2013). For all diseases, ranking was as follows; 1-3 = Resistant: No visible symptoms or very light symptoms; 4-6 = Intermediate: Visible and conspicuous symptoms resulting only in limited economic damage; 7-9 = Susceptible: Severe to very severe symptoms causing considerable yield losses or plant deaths. For ALS, disease symptoms are characterized by round lesions which usually appear as brown spots confined to tissues around veins giving an angular appearance. For CBB, symptoms initially appear as water soaked spots on the underside of the leaves which enlarge and merge to form large brown irregular lesions surrounded by a narrow yellow zone. For rust, spores are present that easily rub off from leafspots that enlarge to form reddish brown pustules (CIAT, 1987; Buruchara, Mukankusi, \& Ampofo, 2010).

\subsection{Data Analysis}

Data for each trial were analyzed separately using linear mixed models in GenStat (Release 16.2, PC/Windows 7; VSN International Ltd., 2013) to assess within trial variability before performing combined analysis of variance (ANOVA) using unbalanced designs option. Genotype (line) mean data were used to determine $\mathrm{G} \times \mathrm{E}$ interactions in Breeding View Standalone statistical tool available in Breeding Management System (IBP, 2013). Stability or lack of phenotypic plasticity for yield was calculated for each genotype using Cultivar Superiority (CS) and Wricke's Ecovalence (WE) stability coefficients. According to Lin and Binns (1988) CS is the sum of the squares of the difference between the genotypic mean in each environment and the mean of the best genotype, divided by twice the number of environments. Genotypes with the smallest values of CS tend to be more stable, and closer to the best genotype in each environment. Wricke (1962) calculated stability as the contribution of each genotype to the genotype-by-environment sum of squares, in an un-weighted analysis of the genotype-by-environment means. A low WE indicates that the genotype responds in a consistent manner to changes in environment; WE does not account for genotype performance. The interactions for $\mathrm{G} \times \mathrm{E}$ were examined for each individual trait using the Additive Main Effects and Multiplicative Interaction (AMMI), and the Genotype Main Effects and Genotype $\times$ Environment Interaction Effects (GGE) Model. In the AMMI model, a two-way ANOVA additive model is performed (additive main effects), followed by a principal component analysis on the residuals (multiplicative interaction). As a result, the interaction is characterized by Interaction Principal Components (IPCA), where genotypes and environments can be simultaneously plotted in biplots. In the GGE model, a 1-way ANOVA, including environment as a main effect, was run followed by a principal component analysis on the residuals (Yan, Hunt, Sheng, \& Szlavnics, 2000; Yan \& Kang, 2003). Like AMMI, principal component scores can be used to construct biplots. In contrast, the genotypic main effects are also represented in the plot in GGE. The GGE model is superior to the AMMI model at differentiating mega-environments (Yan, Kang, Ma, Woods, \& Cornelius, 2007). 


\section{Results}

\subsection{Analysis of Variance (ANOVA)}

The effect of location (L) was not significantly different for most variables in all the six groups of lines except, for some groups, in rust (RUST), plant vigor $(P L N T V I G)$ and days to flowering $(D F)(\mathrm{P} \leq 0.05)$ (Table 1). In general, seasons within locations (Season/L), and replications within season and location (Rep/Season/L) were significantly different $(\mathrm{P} \leq 0.05)$ for most variables. The lines were significantly different $(\mathrm{P} \leq 0.05)$ in a very few variables in four groups; in DAB Set2 none were significantly different and in DAB Set4 all showed significant differences. The interaction of line by season within location (Line $\times$ Season/L) was significant for all groups in some variables. Due to the observed variability in the performance of lines across trials (Line $\times$ Season/L), each trial was considered as a different environment for the Genotype $\times$ Environment Interaction analysis.

Table 1. Analysis of variance for assessed variables at two locations (L) during two seasons, April-July and September-December ${ }^{\#}$

\begin{tabular}{|c|c|c|c|c|c|c|c|c|}
\hline Source of variation & d.f. & $A L S$ & $C B B$ & $R U S T$ & PLNTVIG & $D F$ & $D P M$ & $Y D H A$ \\
\hline \multicolumn{9}{|l|}{$D A B$ Set2 } \\
\hline Location (L) & 1 & 81.05 & 0.06 & $1367.74 *$ & 43.96 & 0.0 & 0.0 & 17519221.0 \\
\hline Season/L & 2 & 85.48 & $327.64 * * *$ & 28.50 & 24.20 & $6383.0 * * *$ & $36965.7 * * *$ & $79875447.0^{* *}$ \\
\hline Rep/Season/L & 4 & $38.40 * * *$ & $5.10 * * *$ & $5.60 * * *$ & $9.68 * * *$ & $40.2 * * *$ & $10.0^{* *}$ & $3914515.3 * * *$ \\
\hline Line & 185 & 0.98 & 1.46 & 1.29 & 1.04 & 13.3 & 8.9 & 474920.6 \\
\hline Line $\times \mathrm{L}$ & 185 & 0.95 & $1.22 *$ & $1.09 * * *$ & $0.91 *$ & 0.0 & 0.0 & 380819.3 \\
\hline Line $\times$ Season $/ \mathrm{L}$ & 370 & $0.83 * *$ & 0.94 & 0.69 & 0.69 & 5.3 & $3.4 * * *$ & $367892.3 * * *$ \\
\hline Residual & 740 & 0.67 & 0.95 & 0.66 & 0.69 & 4.7 & 2.3 & 244887.7 \\
\hline Total & 1487 & 1.05 & 1.49 & 1.77 & 0.84 & 14.0 & 52.8 & 449614.7 \\
\hline \multicolumn{9}{|l|}{$D A B$ Set 4} \\
\hline Location (L) & 1 & 90.03 & 4.85 & $671.63^{* *}$ & 2.42 & $32447.0 *$ & 126017.3 & 8000432.0 \\
\hline Season/L & 2 & $125.62 *$ & $195.14^{*}$ & 3.90 & 26.93 & $967.3 * * *$ & $15241.2 * * *$ & 14502218.0 \\
\hline Rep/Season/L & 4 & $7.97 * * *$ & $13.95 * * *$ & $3.72 * * *$ & $13.70 * * *$ & 15.4 & 8.1 & $9875964.0 * * *$ \\
\hline Line & 95 & $1.35^{* * *}$ & $1.94 * *$ & $2.66 * *$ & $1.42 * * *$ & $31.2 * * *$ & $26.1 * *$ & $382034.0^{*}$ \\
\hline Line $\times \mathrm{L}$ & 95 & 0.50 & 1.10 & $1.42^{* *}$ & 0.73 & 12.6 & 14.6 & 269059.7 \\
\hline Line $\times$ Season $/ L$ & 190 & $0.75 * *$ & $1.13 * * *$ & 0.85 & $0.63 * *$ & $14.1 * * *$ & $14.9 * * *$ & 240558.4 \\
\hline Residual & 380 & 0.55 & 0.74 & 0.72 & 0.47 & 8.3 & 9.8 & 211654.7 \\
\hline Total & 767 & 1.18 & 1.61 & 1.98 & 0.80 & 57.9 & 217.7 & 344846.8 \\
\hline \multicolumn{9}{|l|}{ Black beans } \\
\hline Location (L) & 1 & 13.344 & 4.8679 & 80.4622 & $37.01 *$ & 2878.96 & 20986.47 & 1672376 \\
\hline Season/L & 2 & $80.42 * * *$ & $176.05 * * *$ & $24.15^{* *}$ & 1.38 & $829.0 * * *$ & $4526.9 * * *$ & 9232468.0 \\
\hline Rep/Season/L & 4 & 1.20 & 0.85 & 0.49 & $2.59 * * *$ & 9.0 & $30.5^{* *}$ & $1757977.8^{* * *}$ \\
\hline Line & 33 & 2.58 & 0.96 & 2.06 & $1.44^{*}$ & 54.4 & 26.1 & 650671.4 \\
\hline Line $\times \mathrm{L}$ & 33 & 1.48 & 0.68 & $2.32 * * *$ & 0.75 & 32.3 & 17.0 & 394203.4 \\
\hline Line $\times$ Season $/ \mathrm{L}$ & 66 & $1.60 * * *$ & $1.13 * *$ & 0.90 & 0.47 & 41.5 & $16.2 * * *$ & $480620.3 *$ \\
\hline Residual & 132 & 0.59 & 0.62 & 0.84 & 0.43 & 34.7 & 8.1 & 314209.6 \\
\hline Total & 271 & 1.83 & 2.11 & 1.65 & 0.77 & 54.5 & 124.4 & 497589.3 \\
\hline \multicolumn{9}{|l|}{ Colored beans } \\
\hline Location (L) & 1 & 48.4088 & 0.0137 & 1.469 & 0.0041 & 321.167 & 11235.17 & 3087454 \\
\hline Season/L & 2 & $44.36^{* * *}$ & $60.95 * *$ & 13.51 & 5.20 & $6351.2 * * *$ & $1179.6 * * *$ & $5892513.0 * *$ \\
\hline Rep/Season/L & 4 & 0.23 & $1.77^{*}$ & $3.44 *$ & $1.95^{*}$ & $26.1 * *$ & 4.0 & 279007.3 \\
\hline Line & 15 & $1.89^{*}$ & 0.87 & 4.93 & 2.43 & 30.6 & $27.1 * *$ & $840892.5^{* *}$ \\
\hline Line $\times \mathrm{L}$ & 15 & 0.57 & 0.45 & 2.83 & 1.33 & $19.2 *$ & 5.6 & $235257.0^{*}$ \\
\hline Line $\times$ Season $/ \mathrm{L}$ & 30 & 1.07 & $1.00 *$ & 1.86 & $1.12^{*}$ & 7.3 & 5.6 & 102609.2 \\
\hline Residual & 60 & 0.81 & 0.50 & 1.14 & 0.60 & 5.9 & 3.6 & 207187.5 \\
\hline Total & 127 & 2.01 & 1.64 & 2.23 & 1.14 & 113.7 & 114.0 & 375120.3 \\
\hline \multicolumn{9}{|l|}{ Assorted } \\
\hline Location (L) & 1 & 112.73 & 0.58 & 2.64 & 23.29 & $9562.6^{*}$ & 30238.4 & 6897162.0 \\
\hline Season/L & 2 & $34.18^{*}$ & $382.32 * * *$ & 7.22 & 3.26 & $458.7 * *$ & $6005.5^{* * *}$ & $28495720.5^{*}$ \\
\hline Rep/Season/L & 4 & $2.5^{* *}$ & $3.72 * *$ & $1.17 *$ & $1.59 *$ & $16.8^{*}$ & 13.2 & $3647386.0 * * *$ \\
\hline Line & 55 & 1.67 & 1.71 & $1.20 *$ & 1.36 & $41.2 * * *$ & $31.2 *$ & 1297188.1 \\
\hline Line $\times \mathrm{L}$ & 55 & $1.80 * *$ & 1.28 & 0.71 & 1.00 & $17.5^{*}$ & 18.8 & $875087.1 *$ \\
\hline Line $\times$ Season $/ \mathrm{L}$ & 107 & 0.91 & $1.22 *$ & $0.78 * * *$ & $0.80 *$ & $12.0 * * *$ & $13.1 *$ & 567365.7 \\
\hline Residual & 214 & 0.83 & 0.88 & 0.43 & 0.56 & 6.2 & 9.2 & 454190.5 \\
\hline Total & 438 & 1.50 & 2.85 & 0.69 & 0.84 & 36.8 & 108.6 & 806007.6 \\
\hline
\end{tabular}




\begin{tabular}{|c|c|c|c|c|c|c|c|c|}
\hline \multicolumn{9}{|l|}{ Small red beans } \\
\hline Location (L) & 1 & 284.14 & 0.08 & 63.71 & 24.49 & 9226.0 & 79078.1 & 15934789.0 \\
\hline Season/L & 2 & $378.9 * * *$ & $233.5^{*}$ & $30.4 *$ & 4.55 & $1412.7 * * *$ & $16060.5^{* * *}$ & 3951428.5 \\
\hline Rep/Season/L & 4 & 3.01 & $14.6 * * *$ & $3.3 * *$ & $3.5 * * *$ & 4.8 & $33.2 * * *$ & $1206240.0^{*}$ \\
\hline Line & 79 & 1.60 & 1.59 & 1.26 & 1.26 & $29.1 * *$ & $11.2 *$ & $605951.0 *$ \\
\hline Line $\times \mathrm{L}$ & 79 & 1.94 & 1.13 & $1.2 * *$ & 0.96 & 14.5 & 7.6 & 389972.2 \\
\hline Line $\times$ Season $/ \mathrm{L}$ & 158 & 1.80 & $1.3 * *$ & 0.80 & 0.70 & $13.5^{* * *}$ & 6.5 & 421310.8 \\
\hline Residual & 316 & 1.59 & 0.87 & 0.77 & 0.73 & 8.8 & 5.4 & 393228.1 \\
\hline Total & 639 & 3.32 & 1.90 & 1.10 & 0.88 & 32.0 & 180.8 & 466616.2 \\
\hline
\end{tabular}

Note. ${ }^{\#}$ d. $f=$ degree of freedom, Rep $=$ replication, $A L S=$ angular leaf spot, $C B B=$ common bacterial blight, $P L N T V I G=$ plant vigor, $D F=$ days to $50 \%$ flowering, $D P M=$ days to physiological maturity, $Y D H A=$ clean yield estimated in $\mathrm{kg} \mathrm{ha}^{-1} . * * *, * * *=$ significant at $\mathrm{P} \leq 0.05, \mathrm{P} \leq 0.01$ and $\mathrm{P} \leq 0.001$ respectively.

\subsection{Field Disease Occurrence}

Angular leaf spot $(A L S)$ and common bacterial blight $(C B B)$ were the major diseases in all seasons and locations (Figure 1). A wide range of scores was observed but means were generally low (1.0-5.8) implying that most of the lines had low ratings; however some lines had severe symptoms (rating > 7.0). Also for $C B B$ and $A L S$, a trend suggests a higher disease pressure at Kachwekano than Kawanda. Kachwekano also had an outbreak of Ascochyta blight but its ratings were generally low and are not presented. Among DAB Set2, $A L S, C B B$ and RUST scores ranged from 1 to 5, 1-7 and 1-6, respectively, and for DAB Set4 from 1 to 5, 1-8.1 and 1-6.5; for Black and Colored beans, 1.4-7, 1-7 and 1-8, and 2-7.1 , 1.5-6.1 and 1.0-5.8; and for the Assorted and Small-red beans, 1-6, 1.5-9 and 1-6.5, and 2-8, 2-7 and 1-6 (Figure 1).

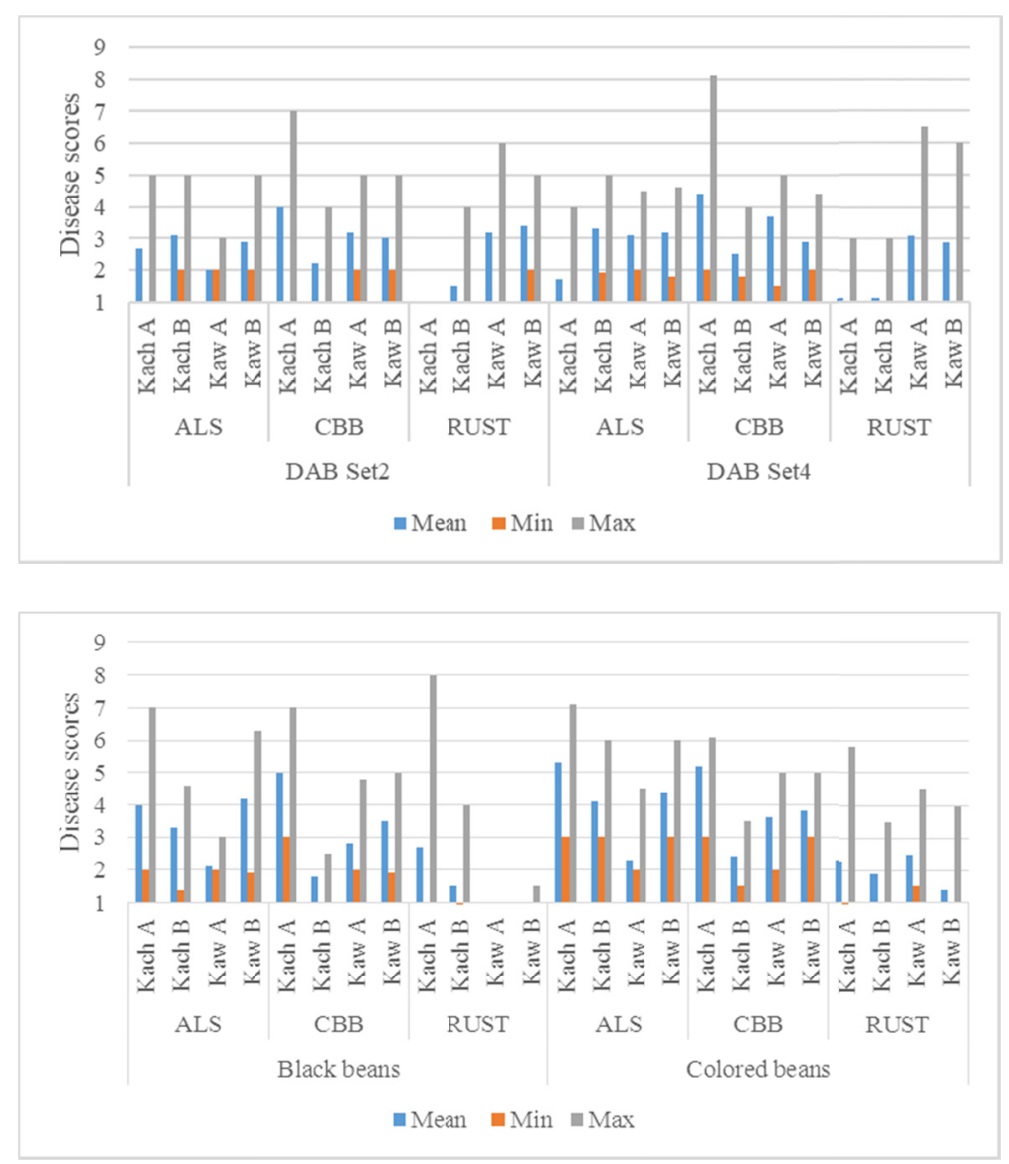




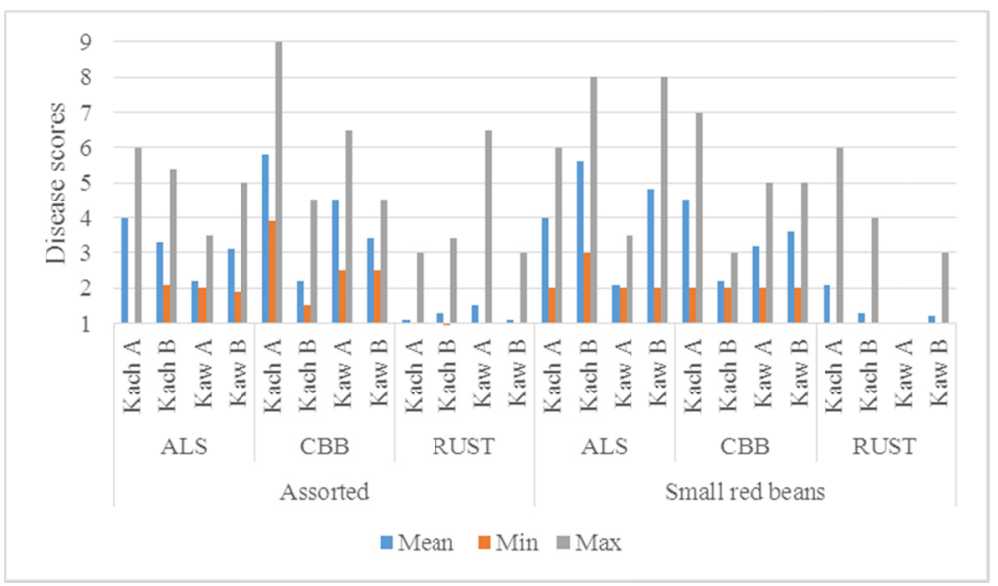

Figure 1. Variation in field diseases at Kawanda and Kachwekano during the first (A) and second (B) season Note. Kach $=$ Kachwekano, Kaw $=$ Kawanda, $A L S F=$ Angular leaf spot in field, $C B B F L=$ common bacterial blight on leaves in field, $R U S T F L=$ Rust on leaves in field.

For $A L S$ and for each group, the lines DAB335, DAB603, SEN116, SIN524, SCR64 and DAD8 had the lowest scores whereas DAB444, DAB940, SEN107, SIN526, SIN526, DAA7 had the highest; 0.6\% (DAB Set2), 38.5\% (DAB Set4), 57.6\% (Black beans), 26.7\% (Colored beans), 74.7\% (Small red beans) and 3.6\% (Assorted) of the lines responded better than CAL96, the susceptible check (Table 2). For $C B B$, the lowest and highest scores were for lines DAB287, DAB603, SCN16, SIN527, SER349, DAD29, and DAB252, DAB932, SCN20, SEC34, SER345, DAA6, respectively; in Black and Small-red beans all lines had lower scores than CAL96, and for DAB Set2, DAB Set4, Colored and Assorted groups, 13.5\%, 87.5\%, 86.7 and 80.0\%, respectively. For RUST, the lowest and highest scores were for lines DAB252, DAB589, SCN18, SEC39, SCR39, DAD36, and DAB351, DAB900, SEN111, SEC42, SCR71, GRR12, respectively; 3.9\% (DAB Set2), 70.8\% (DAB Set4), 24.2\% (Black beans), 53.3\% (Colored beans), $0 \%$ (Small red beans) and $0 \%$ (Assorted) of the lines performed better than CAL96 in the three diseases (Table 2).

Table 2. Disease mean scores for test lines in two locations during two seasons, April-July and September-December

\begin{tabular}{|c|c|c|c|c|c|c|c|c|c|c|c|c|}
\hline & \multicolumn{2}{|l|}{ DAB Set2 } & \multicolumn{2}{|l|}{ DAB Set4 } & \multicolumn{2}{|c|}{ Black beans } & \multicolumn{2}{|c|}{ Colored beans } & \multicolumn{2}{|c|}{ Small red beans } & \multicolumn{2}{|l|}{ Assorted } \\
\hline \multirow{17}{*}{ Angular leaf spot } & DAB335 & 1.8 & DAB603 & 1.8 & SEN116 & 2.1 & SIN524 & 2.8 & SCR64 & 3.0 & DAD8 & 2.0 \\
\hline & DAB270 & 2.0 & DAB932 & 2.0 & SCN15 & 2.2 & SIN527 & 3.3 & SER377 & 3.3 & GGB4 & 2.1 \\
\hline & DAB287 & 2.0 & DAB582 & 2.0 & SEN119 & 2.6 & SEC40 & 3.5 & SER345 & 3.3 & DAD9 & 2.3 \\
\hline & DAB324 & 2.0 & DAB573 & 2.0 & SEN120 & 2.8 & SEC39 & 3.6 & SCR39 & 3.5 & DAD11 & 2.6 \\
\hline & DAB358 & 2.0 & DAB921 & 2.0 & SEN114 & 2.9 & SIN525 & 3.7 & SCR54 & 3.5 & DAA3 & 2.8 \\
\hline & DAB364 & 2.0 & DAB570 & 2.1 & SCN14 & 2.9 & SEC37 & 3.8 & SCR70 & 3.5 & DAD21 & 2.8 \\
\hline & DAB387 & 2.0 & DAB574 & 2.2 & SEN112 & 2.9 & SEC35 & 3.9 & SCR47 & 3.5 & DAD39 & 2.8 \\
\hline & DAB414 & 2.0 & DAB581 & 2.2 & SCN16 & 3.0 & $\mathrm{SEC} 43$ & 3.9 & SEF15 & 3.5 & DAD6 & 2.8 \\
\hline & DAB233 & 3.5 & DAB583 & 3.6 & SCN18 & 4.0 & SEC38 & 4.0 & SER342 & 4.8 & GRR 30 & 3.7 \\
\hline & DAB316 & 3.5 & DAB915 & 3.6 & SEN101 & 4.0 & $\mathrm{SEC} 42$ & 4.1 & SCR57 & 4.9 & GRR 25 & 3.8 \\
\hline & DAB355 & 3.5 & SEQ1003 & 3.6 & $\mathrm{SCN} 20$ & 4.0 & SEC33 & 4.3 & SCR71 & 4.9 & GRR 5 & 3.8 \\
\hline & DAB470 & 3.5 & DAB909 & 3.6 & SEN109 & 4.1 & SEC41 & 4.4 & SCR49 & 5.0 & DAA9 & 3.9 \\
\hline & DAB476 & 3.5 & DAB942 & 3.6 & NCB280 & 4.4 & SEC34 & 4.5 & SCR69 & 5.0 & GGB6 & 3.9 \\
\hline & DAB351 & 3.7 & DAB941 & 3.7 & SEN103 & 4.5 & SEC36 & 5.0 & SER352 & 5.0 & GGB8-2 & 4.0 \\
\hline & DAB444 & 3.8 & DAB940 & 4.0 & SEN107 & 4.6 & SIN526 & 5.2 & SER363 & 5.3 & DAA7 & 4.4 \\
\hline & CAL96 & 2.0 & CAL96 & 2.7 & CAL96 & 3.5 & CAL96 & 3.6 & CAL96 & 4.3 & CAL96 & 2.2 \\
\hline & $<C A L 96^{\#}$ & $1 \%$ & & $39 \%$ & & $58 \%$ & & $27 \%$ & & $75 \%$ & & $4 \%$ \\
\hline \multirow{5}{*}{$\begin{array}{l}\text { Common } \\
\text { bacterial blight }\end{array}$} & DAB287 & 1.8 & DAB603 & 2.3 & SCN16 & 2.5 & SIN527 & 3.0 & SER349 & 2.0 & DAD29 & 2.8 \\
\hline & DAB378 & 2.0 & DAB602 & 2.4 & SCN15 & 2.6 & SEC33 & 3.1 & SCR60 & 2.2 & GGB1 & 2.9 \\
\hline & DAB540 & 2.0 & DAB914 & 2.4 & SEN109 & 2.8 & SIN524 & 3.3 & SCR44 & 2.5 & GRR 25 & 3.1 \\
\hline & DAB324 & 2.3 & DAB584 & 2.5 & SEN122 & 2.9 & SIN525 & 3.3 & SER348 & 2.5 & DAD34 & 3.3 \\
\hline & DAB359 & 2.3 & SAB659 & 2.6 & NCB280 & 2.9 & SEC40 & 3.4 & SCR61 & 2.6 & GRR 21 & 3.4 \\
\hline
\end{tabular}




\begin{tabular}{|c|c|c|c|c|c|c|c|c|c|c|c|c|}
\hline \multirow{12}{*}{$\begin{array}{l}\text { Common } \\
\text { bacterial blight }\end{array}$} & DAB364 & 2.3 & DAB380 & 2.6 & SEN104 & 3.0 & SEC42 & 3.5 & SER366 & 2.7 & GGB6 & 3.4 \\
\hline & DAB413 & 2.3 & DAB909 & 2.7 & SEN110 & 3.1 & SIN526 & 3.5 & SER360 & 2.7 & DAD22 & 3.5 \\
\hline & DAB496 & 2.3 & DAB574 & 2.7 & $\mathrm{SCN} 21$ & 3.1 & SEC41 & 3.7 & SER335 & 2.7 & DAD23 & 3.5 \\
\hline & DAB348 & 4.0 & DAB931 & 4.2 & SEN107 & 3.5 & SEC 38 & 3.7 & SER354 & 4.0 & DAA8 & 4.6 \\
\hline & DAB349 & 4.0 & DAB925 & 4.3 & SEN108 & 3.5 & SEC37 & 3.8 & SEF14 & 4.0 & GRR 16 & 4.6 \\
\hline & DAB474 & 4.0 & DAB923 & 4.3 & SEN111 & 3.6 & SEC43 & 3.9 & SER326 & 4.0 & DAD38 & 4.8 \\
\hline & DAB315 & 4.3 & DAB905 & 4.4 & SEN115 & 3.6 & SEC36 & 4.0 & SEF 29 & 4.1 & DAA10 & 4.9 \\
\hline & DAB478 & 4.3 & DAB929 & 4.5 & SEN117 & 3.6 & SEC35 & 4.0 & SCR47 & 4.1 & DAD49 & 4.9 \\
\hline & DAB314 & 5.0 & DAB941 & 4.5 & SEN121 & 3.6 & SEC39 & 4.2 & SER357 & 4.3 & GRR 26 & 4.9 \\
\hline & DAB252 & 6.0 & DAB932 & 4.9 & SCN20 & 3.9 & SEC34 & 4.2 & SER345 & 4.5 & DAA6 & 5.5 \\
\hline & CAL96 & 2.8 & CAL96 & 4.0 & CAL96 & 4.6 & CAL96 & 4.1 & CAL96 & 4.7 & CAL96 & 4.3 \\
\hline & $<C A L 96$ & $14 \%$ & & $88 \%$ & & $100 \%$ & & $87 \%$ & & $100 \%$ & & $80 \%$ \\
\hline \multirow{17}{*}{ Rust } & DAB252 & 1.0 & DAB589 & 1.1 & SCN18 & 1.0 & SEC39 & 1.1 & SCR39 & 1.0 & DAD36 & 1.0 \\
\hline & DAB314 & 1.0 & DAB588 & 1.2 & SEN103 & 1.0 & SEC37 & 1.3 & SCR45 & 1.0 & DAD40 & 1.0 \\
\hline & DAB219 & 1.5 & DAB574 & 1.3 & SEN106 & 1.0 & SEC40 & 1.3 & SCR46 & 1.0 & GRR 30 & 1.0 \\
\hline & DAB251 & 1.5 & SAB659 & 1.4 & SEN118 & 1.0 & SEC34 & 1.5 & SCR51 & 1.0 & GRR 5 & 1.0 \\
\hline & DAB293 & 1.5 & DAB579 & 1.4 & SCN14 & 1.0 & SEC35 & 1.5 & SCR55 & 1.0 & GRR 18 & 1.0 \\
\hline & DAB474 & 1.5 & DAB601 & 1.4 & SEN119 & 1.0 & SIN527 & 1.5 & SCR74 & 1.0 & GRR 23 & 1.0 \\
\hline & DAB218 & 1.8 & DAB572 & 1.5 & SEN101 & 1.1 & SEC38 & 1.5 & SCR60 & 1.0 & DAA1 & 1.1 \\
\hline & DAB221 & 1.8 & DAB569 & 1.5 & SEN114 & 1.1 & SIN526 & 1.7 & SEF 16 & 1.0 & DAA3 & 1.1 \\
\hline & DAB491 & 3.3 & DAB924 & 3.1 & SCN15 & 2.0 & SEC 36 & 2.1 & SER357 & 2.0 & DAD32 & 1.7 \\
\hline & DAB483 & 3.3 & DAB915 & 3.1 & SEN102 & 2.0 & SEC33 & 2.5 & SCR65 & 2.3 & DAA6 & 1.7 \\
\hline & DAB515 & 3.3 & DAB926 & 3.2 & SEN113 & 2.0 & SEC41 & 2.8 & SER355 & 2.3 & DAD39 & 1.8 \\
\hline & DAB526 & 3.3 & DAB933 & 3.4 & NCB280 & 2.1 & SIN524 & 2.8 & SER373 & 2.3 & GRR 8 & 2.1 \\
\hline & DAB313 & 3.7 & DAB906 & 3.5 & $\mathrm{SCN} 20$ & 2.2 & SEC43 & 3.1 & SCR50 & 2.5 & GRR 25 & 2.1 \\
\hline & DAB347 & 3.7 & DAB903 & 3.7 & NCB226 & 2.3 & SIN525 & 3.2 & SER366 & 2.5 & DAD38 & 2.3 \\
\hline & DAB351 & 3.7 & DAB900 & 4.5 & SEN111 & 3.5 & SEC42 & 3.3 & SCR71 & 2.8 & GRR 12 & 2.9 \\
\hline & CAL96 & 1.8 & CAL96 & 2.3 & CAL96 & 1.2 & CAL96 & 1.8 & CAL96 & 1.0 & CAL96 & 1.0 \\
\hline & $<C A L 96$ & $4 \%$ & & $71 \%$ & & $24 \%$ & & $53 \%$ & & $0 \%$ & & $0 \%$ \\
\hline
\end{tabular}

Note. ${ }^{\#}<$ CAL96 percentage of lines that responded to diseases better than CAL96 across environments.

\subsection{Yield Related Traits}

\subsubsection{Analysis of Variance for AMMI Model}

Replication means for each trial were analyzed. Each location (Kachwekano, Kawanda) and season (A, B) was considered as different environments. Significant differences $(\mathrm{P} \leq 0.05)$ among lines were found in plant vigor, $D F, D P M$ and $Y D H A$ for DAB Set4, Assorted, Red and Colored beans (Table 3). Environments were highly different $(\mathrm{P} \leq 0.001)$ for all the variables measured in six groups. Among DAB Set4, significant $(\mathrm{P} \leq 0.05)$ IPCA 1 and IPCA 2 were obtained in all variables; for the Black and Red beans, both IPCA 1 and IPCA 2 were significant $(\mathrm{P} \leq 0.05)$ for $D F$ and $D P M$. In addition, IPCA 1 was significant for $Y D H A$ in the Black beans and plant vigor in the Red beans. In the Assorted beans, both IPCA 1 and IPCA 2 were significant for plant vigor and $D P M$. Among DAB Set2 and Colored beans, IPCA 1 was significant for plant vigor and YDHA (Table 3). 
Table 3. Variance and genotype by environment $(\mathrm{G} \times \mathrm{E})$ interaction for AMMI model for days to flowering (DF) and maturity (DPM) and clean yield (YDHA)

\begin{tabular}{|c|c|c|c|c|c|c|c|c|c|c|}
\hline Source of variation & d.f. & Plant vigor & $D F$ & $D P M$ & $Y D H A$ & d.f. & Plant vigor & $D F$ & $D P M$ & $Y D H A$ \\
\hline & \multicolumn{5}{|c|}{ DAB Set4 } & \multicolumn{5}{|c|}{ Black beans } \\
\hline Genotypes & 96 & $0.73 * * *$ & $16.0 * * *$ & $13.0 * * *$ & $206425^{* *}$ & 33 & $0.71 * * *$ & 23.4 & 10.9 & 318188 \\
\hline Environments & 3 & $9.72 * * *$ & $6030.0 * * *$ & $28205.0 * * *$ & $7326482 * * *$ & 3 & $6.16^{* * *}$ & $769.0 * * *$ & $5130.8 * * *$ & $3396629 * * *$ \\
\hline Interactions & 288 & 0.34 & 7.0 & 8.0 & 134927 & 99 & 0.29 & 18.7 & 8.1 & 241684 \\
\hline IPCA 1 & 98 & $0.44 * * *$ & $14.0 * * *$ & $13.0 * * *$ & $199938 * * *$ & 35 & 0.35 & $46.8 * * *$ & $15.3 * * *$ & $406608 * * *$ \\
\hline IPCA 2 & 96 & $0.35^{*}$ & $5.0 * * *$ & $7.0^{* * *}$ & $120759^{*}$ & 33 & 0.29 & $5.4 * * *$ & $7.2 * * *$ & 182154 \\
\hline \multirow[t]{2}{*}{ Residuals } & 94 & 0.22 & 1.0 & 3.0 & 81619 & 31 & 0.21 & 1.2 & 0.8 & 118851 \\
\hline & \multicolumn{5}{|c|}{ Assorted } & \multicolumn{5}{|c|}{ Small red beans } \\
\hline Genotypes & 55 & $0.68 *$ & $20.7 * * *$ & $16.0 * * *$ & $652455^{* *}$ & 79 & $0.81 * * *$ & $14.5^{* * *}$ & $6.0^{* *}$ & $306778^{*}$ \\
\hline Environments & 3 & $4.90 * * *$ & $1765.7 * * *$ & $7320.0 * * *$ & $11760952 * * *$ & 3 & $4.53 * * *$ & $2039.0^{* * *}$ & $19450.0 * * *$ & $4223137 * * *$ \\
\hline Interactions & 165 & 0.44 & 6.9 & 8.0 & 359300 & 237 & 0.46 & 6.9 & 4.0 & 207105 \\
\hline IPCA 1 & 57 & $0.53 *$ & $15.0 * * *$ & $11.0^{* *}$ & $498261 * *$ & 81 & $0.58 *$ & $15.3 * * *$ & $6.0 * * *$ & 245293 \\
\hline IPCA 2 & 55 & $0.49^{*}$ & $4.0 * * *$ & 8.0 & 345812 & 79 & 0.41 & $3.2^{*}$ & $3.0 * * *$ & 200986 \\
\hline \multirow[t]{2}{*}{ Residuals } & 53 & 0.31 & 1.2 & 5.0 & 223849 & 77 & 0.38 & 1.9 & 1.0 & 173212 \\
\hline & \multicolumn{5}{|c|}{ DAB Set2 } & \multicolumn{5}{|c|}{ Colored beans } \\
\hline Genotypes & 155 & 0.47 & & & 273082 & 15 & $1.32 *$ & & & $414397 *$ \\
\hline Environments & 3 & $10.97 * * *$ & & & $26413385^{* * *}$ & 3 & $2.23 *$ & & & $2848092 * * *$ \\
\hline Interactions & 465 & 0.47 & & & 261935 & 45 & 0.60 & & & 203655 \\
\hline IPCA 1 & 157 & $0.66 * * *$ & & & $415417 * * *$ & 17 & $1.01^{* *}$ & & & $394066^{* *}$ \\
\hline IPCA 2 & 155 & 0.42 & & & $219020^{* *}$ & 15 & 0.45 & & & 104842 \\
\hline Residuals & 153 & 0.34 & & & 147917 & 13 & 0.24 & & & 68671 \\
\hline
\end{tabular}

Note. $d . f=$ degree of freedom, $*, * *, * * *=$ significant at $\mathrm{P} \leq 0.05, \mathrm{P} \leq 0.01$ and $\mathrm{P} \leq 0.001$ respectively.

\subsubsection{Growth Vigor}

Growth vigor was generally better at Kawanda than at Kachwekano with scores ranging from 1 (excellent) to 5 (intermediate) (Figure 2). Majority of the lines in all the groups showed excellent or good vigor (1-4). Intermediate vigor was shown by $7 \%$ of lines in Colored bean group and $1 \%$ of lines in Small red bean group at Kachwekano.

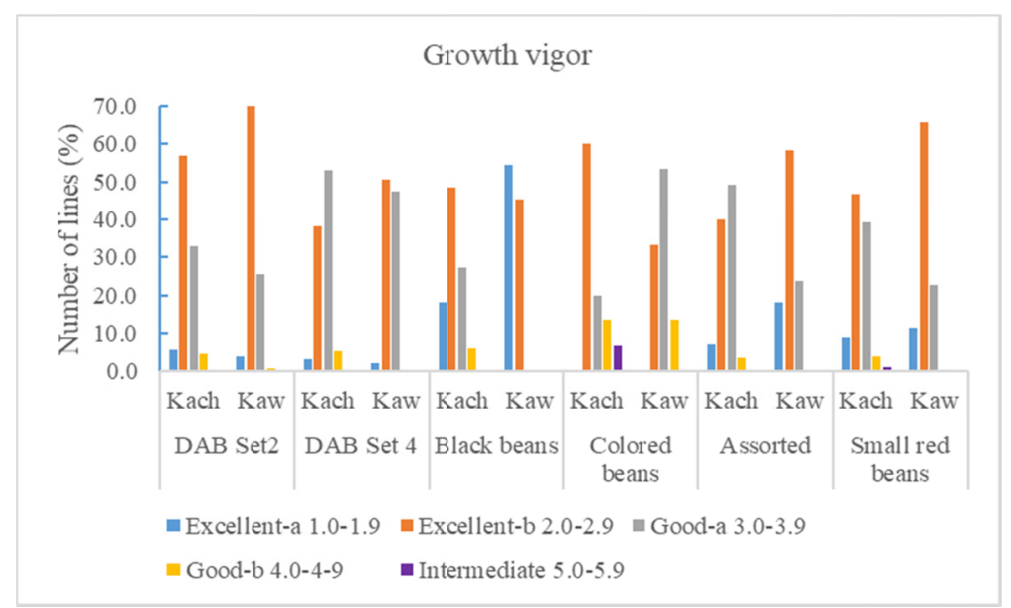

Figure 2. Number of lines (\%) grouped according to 1-9 scale for growth vigor

\subsubsection{Days to Flowering $(D F)$ and Physiological Maturity (DPM)}

These two variables were generally higher at Kachwekano than at Kawanda. $D F$ ranged from 30-46 at Kawanda and 37-58 at Kachwekano whereas DPM ranged from 62-78 (Kawanda) and 82-108 (Kachwekano) (Figure 3). 
For DAB Set4, the earliest maturing lines were 71 (DAB918), 2 (CAL96) and 74 (DAB921) that matured on day 80 and 84 at Kachwekano, and 59 (DAB906), 49 (DAB631) and 83 (DAB930) on day 64 and 65 at Kawanda. DAB918 matured on day 66 at Kawanda thus it showed consistency in early maturity in the data set. Among the black beans, line 1 (CAL96) was the earliest to mature on day 86 followed by 34 (SEN122), 32 (SEN120) and 27 (SEN115) on day 88 at Kachwekano. All these lines matured on day 74 at Kawanda but the earliest lines which matured on day 73 at this location were 3 (NCB280), 4 (SCN13), 30 (SEN118), 5 (SCN14), 12 (SCN21), 14 (SEN102) and 8 (SCN17). In case of Assorted beans, the lines 13 (DAD21), 15 (DAD23), 56 (GRR8) and 1 (CAL96) matured earliest on day 84 or 85 at Kachwekano. Of these CAL96 was among the earliest to mature at Kawanda on day 70 and DAD23 matured on day 72. Among the Small-red beans, 1 (CAL96) and 62 (SER345) matured earliest on day 88 at Kachwekano. At Kawanda, they reached maturity at 73 and 74 days while the earliest to mature was 41 (SEF14) on day 70 (Figure 3).
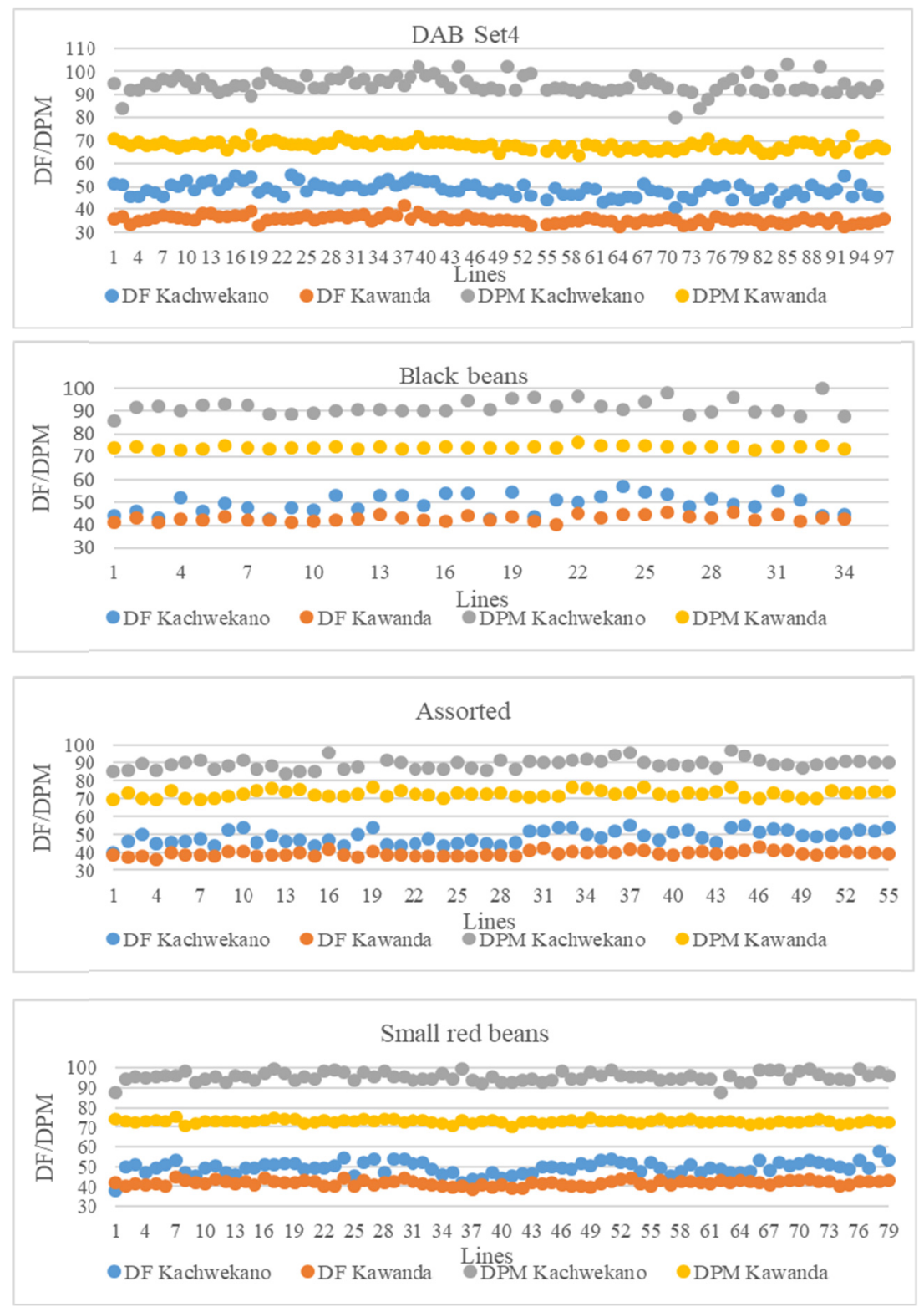

Figure 3. Days to 50\% flowering (DF) and physiological maturity (DPM) at Kawanda and Kachwekano

\subsubsection{Yield Performance}

Yield not only highly varied among groups but also between the two locations and the two seasons they were evaluated in (Figure 4). Generally, DAB Set4 was the poorest yielding group while DAB Set2 and Black beans 
were the best. Kachwekano B recorded the highest mean yields for DAB Set2, Black and Colored beans with ranges of 336-3786 kg ha ${ }^{-1}, 1064-3738 \mathrm{~kg} \mathrm{ha}^{-1}$ and 602-3299 $\mathrm{kg} \mathrm{ha}^{-1}$ respectively. The lowest mean yields for these three groups were obtained at Kachwekano A. DAB Set4, Small red beans and Assorted group obtained the highest mean yields at Kachwekano A, Kawanda B and Kawanda A, with a range of 436-2981 kg ha ${ }^{-1}, 740-2966$ $\mathrm{kg} \mathrm{ha}^{-1}$ and 904-3414 $\mathrm{kg} \mathrm{ha}^{-1}$ respectively (Figure 4).
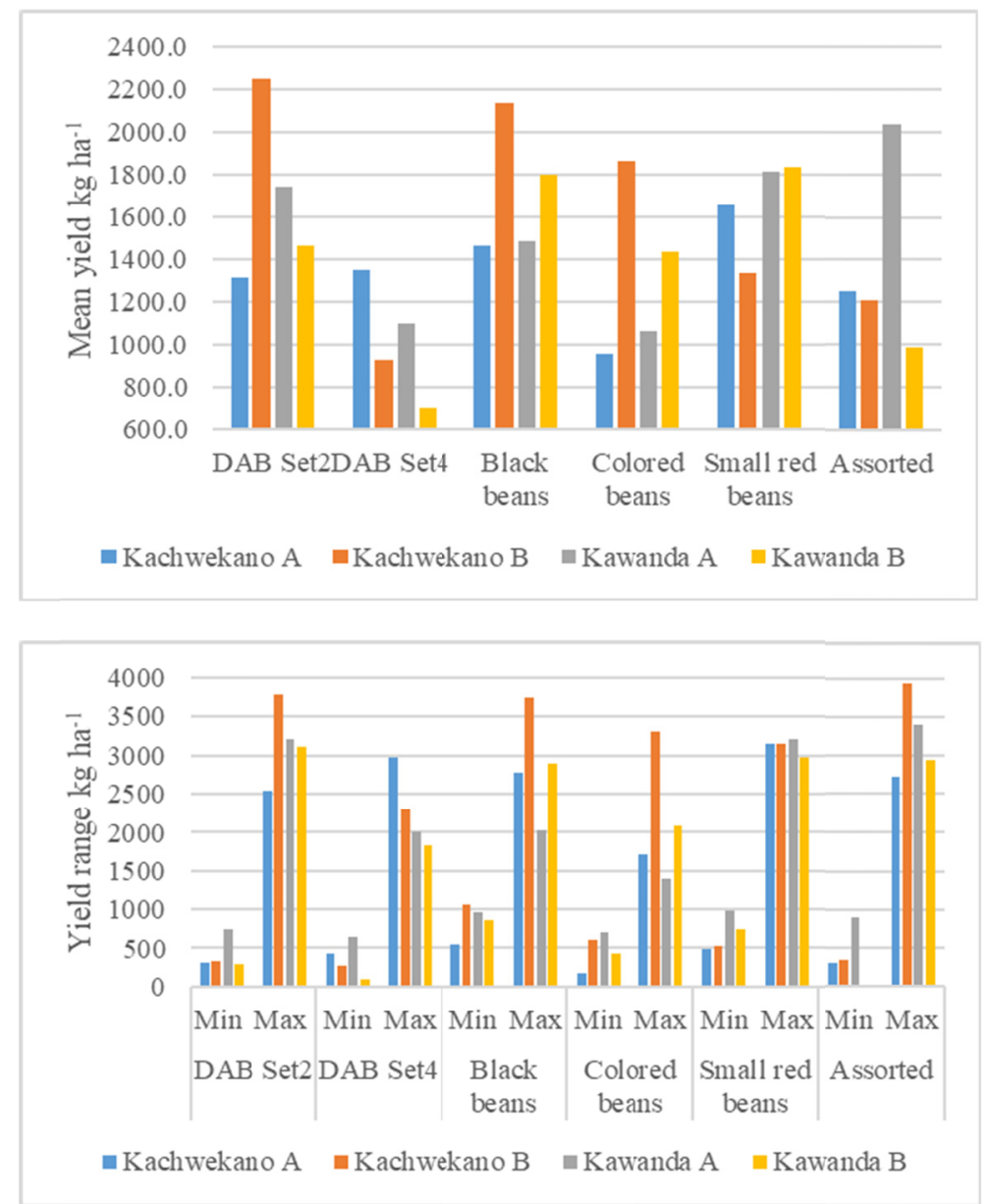

Figure 3. Mean clean yield and range of lines in four environments; $\mathrm{A}=$ first season, $\mathrm{B}=$ second season

\subsubsection{Genotype, Environment Interaction for Yield}

The AMMI biplots reveal patterns of $\mathrm{G} \times \mathrm{E}$ and inform on adaptation of genotypes (lines) to environments basing on $\mathrm{G} \times \mathrm{E}$ and not performance. In this study, all environments except Kawanda A shared fairly equal responsibility for $G \times E$ in DAB Set2. Kachwekano $(A / B)$ was the most responsible for $G \times E$ in DAB Set4 whereas Kawanda B/Kachwekano B was most responsible in Black and Colored beans (Figure 5). For Assorted beans, Kawanda was generally responsible for most $\mathrm{G} \times \mathrm{E}$ but only Kawanda $\mathrm{B}$ was most responsible in Small-red beans. GGE biplot was used to group environments in homogenous sets (mega-environments). For DAB Set2, the GGE analysis did not group any of the four environments into main mega environments for yield (Figure 5). The total variation that could be explained was $66.9 \%$ and majority of the lines are close to the axes signifying stability. Kawanda A/B were the best environments for most of the lines. The lines 84 (DAB389), 6 (DAB222), 39 (DAB299), 33 (DAB287) were some of the high yielding but less stable lines while 5 (DAB221), 32 (DAB282), 83 (DAB288), 22 (DAB254) and 70 (DAB361) were among the relatively high yielding and more stable lines. Yields for DAB Set 4 were grouped into three major mega environments where Kawanda A/B were grouped together while Kachwekano A/B were separate. There was no line recommended for Kachwekano A but several lines were suitable for the other environments. In this group, the total explainable variation was $74.8 \%$. The line 18 (DAB582) was relatively high yielding and stable whereas 96 (SAB659), 92 (DAB939), 30 (DAB601) and 94 (DAB941) were high yielding but less stable. For the Black beans, Kawanda B and 
Kachwekano A were grouped as one and Kawanda A and Kachwekano B were separate. The total explainable variation was $78.8 \%$ and the majority of the lines were away from the axes indicating instability. The line 19 (SEN107) was stable and high yielding. For Colored, Assorted and Small red beans, environments were grouped into two main ones. Among the Colored beans, Kawanda A and Kachwekano B were grouped together and no line was best suited there. The lines 9 (SEC40), 10 (SEC41) were high yielding and suitable for Kawanda B and Kachwekano A. For Assorted beans, Kawanda A/B were together and had more suitable lines (53 (GRR29), 42 (GGB9), 54 (GRR30), 51 (GRR25) than Kachwekano A/B (48 (GRR18), 6 (DAA7). Among Small-red beans, Kawanda A/B, and Kachwekano B were grouped as one main environment while Kachwekano A was alone with no suitable lines. The lines 56 (SER339), 32 (SCR60), 52 (SER335) and 18 (SCR66) were best suited for the former group (Figure 5).
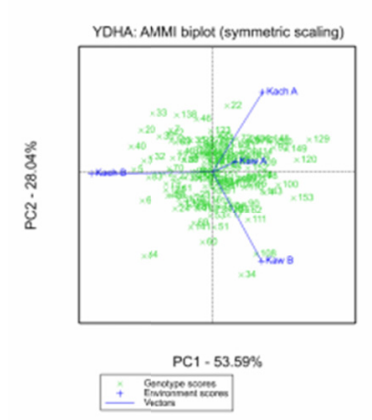

DAB Set2
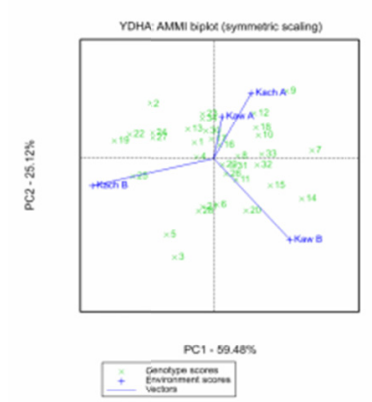

Black beans
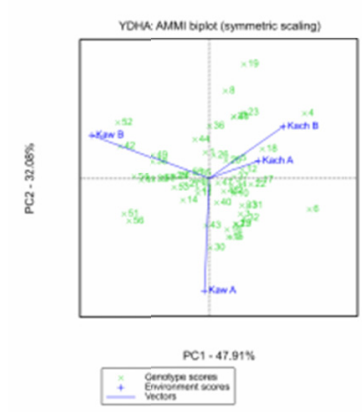

Assorted
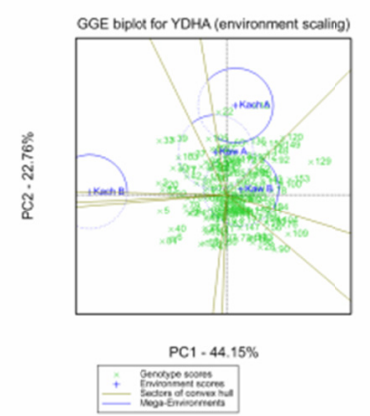

DAB Set2
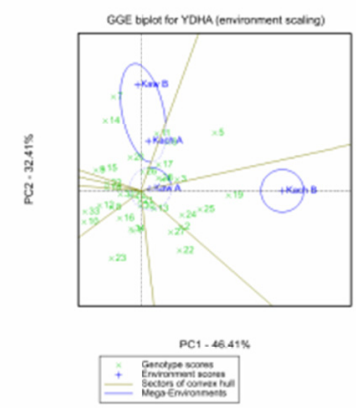

Black beans
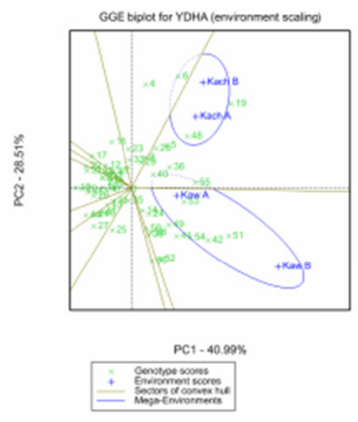

Assorted
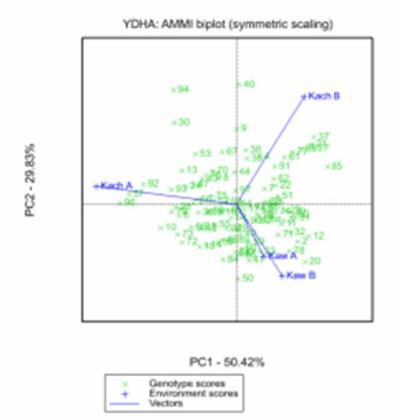

DAB Set4
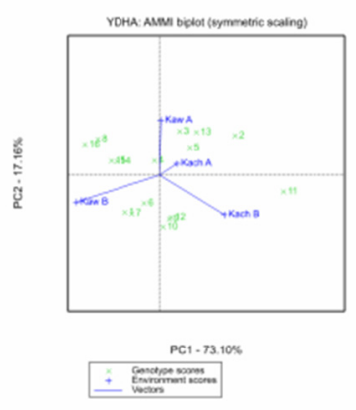

Colored beans
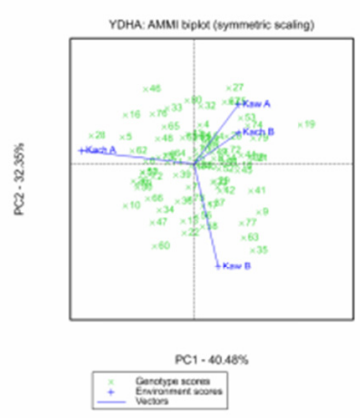

Small red beans
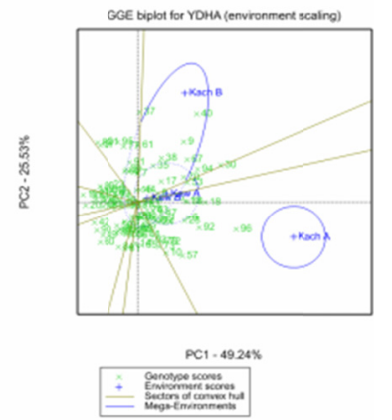

DAB Set4
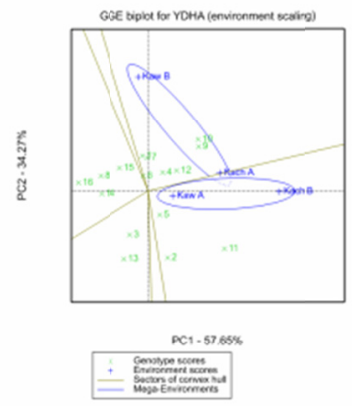

Colored beans
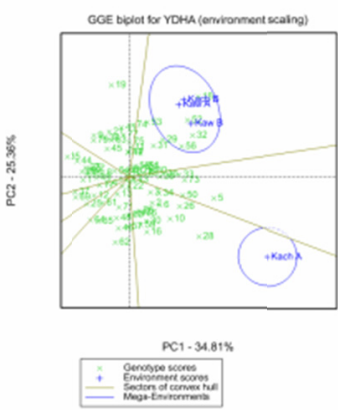

Small red beans

Figure 5. AMMI and GGE biplots for yield of lines from six groups in four environments

\subsubsection{Yield Stability}

Lines were also categorized according to general performance and stability for yield (Table 4). Among DAB Set2, lines DAB299 (2533 $\left.\mathrm{kg} \mathrm{ha}^{-1}\right)$, DAB291 (2202 $\left.\mathrm{kg} \mathrm{ha}^{-1}\right)$, DAB249 and DAB449 combined high performance and consistency considering their low values for cultivar superiority. Based on the Wricke's ecovalence, 
DAB328 (1724 kg ha $\left.{ }^{-1}\right)$, DAB398 (1591 $\left.\mathrm{kg} \mathrm{ha}^{-1}\right)$, DAB315, DAB357, DAB523 and DAB391 were the most stable with respect to changing environments (dynamic stability). In this group, none of the best performers expressed dynamic stability. This observation was also true for DAB Set4. However, among the lines that combined high performance and consistency namely; DAB582 (1700 kg ha $\left.{ }^{-1}\right)$, DAB601 (1571 kg ha $\left.{ }^{-1}\right)$, SAB659, DAB611, DAB571, DAB917, DAB914 and DAB568, three lines including DAB601, SAB659 and DAB611 were among the least stable. The lines DAB901 $\left(1019 \mathrm{~kg} \mathrm{ha}^{-1}\right)$, SEQ1003 $\left(858 \mathrm{~kg} \mathrm{ha}^{-1}\right)$, DAB613 (569 kg ha $\left.{ }^{-1}\right)$, DAB580 and DAB930 showed high stability although their yield performance were generally low. Among the Black beans, three of the high performing and consistent lines namely SCN20 (2167 kg ha $\left.{ }^{-1}\right)$, SCN13 (1873 kg $\left.\mathrm{ha}^{-1}\right)$ and SEN114 (1875 $\left.\mathrm{kg} \mathrm{ha}^{-1}\right)$ also expressed stability. In the same category of high performers, SCN14 (2358 $\left.\mathrm{kg} \mathrm{ha}^{-1}\right)$, SEN107 $\left(2203 \mathrm{~kg} \mathrm{ha}^{-1}\right)$, SEN113 $\left(1868 \mathrm{~kg} \mathrm{ha}^{-1}\right)$ and SEN112 $\left(1844 \mathrm{~kg} \mathrm{ha}^{-1}\right)$ were among the least stable The lines SEN104 (SEN104) and SEN117 (SEN117) had the lowest Wricke's ecovalence and thus are the most stable in the group. In the case of Colored beans, four of the lines that combined high performance and consistency; SEC40 (1860 kg ha $\left.{ }^{-1}\right), \operatorname{SEC} 35\left(1559 \mathrm{~kg} \mathrm{~h}^{-1}\right), \operatorname{SEC} 36\left(1338 \mathrm{~kg} \mathrm{ha}^{-1}\right)$ and SEC37 (1301 kg ha $\left.{ }^{-1}\right)$ also expressed stability. For the Assorted beans, DAD34 (2411 kg ha $\left.{ }^{-1}\right)$, DAA7 $\left(2308 \mathrm{~kg} \mathrm{ha}^{-1}\right)$, GRR18 (1988 kg ha-1), DAA6, GGB2 and DAD44 combined high performance and consistency. None of these lines appeared among the most stable. Among the small red beans, SCR66 (2422 kg ha $\left.{ }^{-1}\right)$, SCR60 (2275 kg ha $\left.{ }^{-1}\right)$, SER335 (2270 kg $\left.\mathrm{ha}^{-1}\right)$ and SCR46 $\left(2249 \mathrm{~kg} \mathrm{ha}^{-1}\right)$ combined high performance with consistency. Of these SCR46 and SCR66 were among the least stable (Table 4).

Table 5. Stability coefficients for selected lines (genotype) by across environment data

\begin{tabular}{|c|c|c|c|c|c|c|c|c|c|c|c|}
\hline Line & Means & $C S$ & $W E$ & Line & Means & $C S$ & $W E$ & Line & Means & $C S$ & $W E$ \\
\hline \multicolumn{4}{|l|}{ DABSet2 } & \multicolumn{4}{|l|}{ DABSet4 } & \multicolumn{4}{|c|}{ Black beans } \\
\hline DAB299 & 2533 & 308194 & 880076 & DAB582 & 1700 & 245430 & 253324 & $\overline{\text { SCN14 }}$ & 2358 & 197981 & 901002 \\
\hline DAB291 & 2202 & 510836 & 211816 & DAB601 & 1571 & 281828 & 604698 & $\mathrm{SCN} 20$ & 2167 & 329981 & 149086 \\
\hline DAB249 & 2161 & 515949 & 227111 & SAB 659 & 1623 & 318499 & 871848 & SEN107 & 2203 & 350274 & 1091170 \\
\hline DAB449 & 2255 & 517919 & 321344 & DAB611 & 1531 & 376008 & 542123 & SCN15 & 2175 & 375854 & 321597 \\
\hline DAB293 & 2134 & 593773 & 451016 & DAB571 & 1440 & 388808 & 272880 & SEN105 & 1939 & 463304 & 328980 \\
\hline DAB443 & 2108 & 661143 & 340523 & DAB917 & 1392 & 397838 & 206871 & SCN13 & 1873 & 501389 & 246215 \\
\hline DAB439 & 2122 & 680882 & 783053 & DAB914 & 1444 & 404442 & 352902 & SEN113 & 1868 & 575104 & 863134 \\
\hline DAB369 & 2071 & 721013 & 724830 & DAB568 & 1361 & 470482 & 382246 & SEN112 & 1844 & 600405 & 497248 \\
\hline DAB234 & 1982 & 738091 & 147898 & DAB610 & 1141 & 775463 & 23178 & SEN114 & 1875 & 624358 & 129647 \\
\hline DAB523 & 1904 & 821281 & 139580 & DAB930 & 1047 & 794195 & 139695 & SEN116 & 1789 & 647340 & 428821 \\
\hline DAB328 & 1724 & 1042236 & 232193 & DAB901 & 1019 & 827791 & 75472 & NCB226 & 1889 & 677841 & 534497 \\
\hline DAB543 & 1775 & 1046820 & 12733 & DAB580 & 971 & 883510 & 117869 & SEN101 & 1720 & 711284 & 195600 \\
\hline DAB398 & 1591 & 1236488 & 188759 & DAB603 & 970 & 1004976 & 20292 & SEN118 & 1668 & 795423 & 84262 \\
\hline DAB270 & 1653 & 1248800 & 23178 & DAB910 & 887 & 1034158 & 9010 & SEN117 & 1637 & 871420 & 97876 \\
\hline DAB359 & 1610 & 1275223 & 27417 & DAB587 & 919 & 1036454 & 4798 & SEN119 & 1523 & 981427 & 91875 \\
\hline DAB235 & 1629 & 1280775 & 16060 & DAB935 & 834 & 1126554 & 5111 & SEN106 & 1562 & 1009231 & 51688 \\
\hline DAB357 & 1556 & 1299987 & 235536 & DAB905 & 780 & 1160660 & 69301 & SEN120 & 1558 & 1032159 & 84295 \\
\hline DAB391 & 1544 & 1313584 & 161747 & DAB589 & 772 & 1206045 & 9157 & SEN122 & 1524 & 1056794 & 108079 \\
\hline DAB315 & 1422 & 1537878 & 194620 & DAB915 & 726 & 1274010 & 15970 & SEN104 & 1444 & 1125591 & 51393 \\
\hline DAB343 & 1395 & 1638055 & 20289 & DAB633 & 680 & 1368424 & 30176 & SCN17 & 1543 & 1171775 & 183932 \\
\hline DAB232 & 1378 & 1678911 & 29703 & DAB937 & 600 & 1465638 & 37469 & $\mathrm{SCN} 21$ & 1483 & 1182308 & 13762 \\
\hline DAB413 & 1248 & 1900610 & 21393 & DAB613 & 569 & 1512307 & 43014 & SEN121 & 1252 & 1514119 & 64322 \\
\hline DAB335 & 1097 & 2207036 & 29967 & AFR 298 & 1149 & 650917 & 238590 & SEN111 & 1214 & 1552921 & 127398 \\
\hline DAB442 & 1091 & 2258157 & 20264 & SEQ1003 & 858 & 1031507 & 83714 & SCN19 & 1270 & 1561726 & 68796 \\
\hline CAL96 & 1884 & 837461 & 373917 & CAL96 & 977 & 1030650 & 43549 & CAL96 & 1382 & 1139594 & 167830 \\
\hline \multicolumn{4}{|c|}{ Colored beans } & \multicolumn{4}{|l|}{ Assorted } & \multicolumn{4}{|c|}{ Small red beans } \\
\hline SEC40 & 1860 & 68797 & 206634 & $\begin{array}{l}\text { DAD34 } \\
\end{array}$ & 2411 & 521304 & 4870541 & $\begin{array}{l}\text { SCR66 } \\
\text { SCR }\end{array}$ & 2422 & 377671 & 1631016 \\
\hline SEC41 & 1874 & 75424 & 361279 & DAA7 & 2308 & 902197 & 3077224 & SCR60 & 2275 & 404037 & 496719 \\
\hline $\mathrm{SEC} 43$ & 1569 & 177883 & 170434 & GRR18 & 1988 & 1018362 & 1342885 & SER335 & 2270 & 450153 & 1131247 \\
\hline SEC35 & 1559 & 276718 & 62279 & DAA6 & 1835 & 1107246 & 304931 & SCR46 & 2249 & 588247 & 1220604 \\
\hline SEC42 & 1603 & 337845 & 2701915 & GRR29 & 1905 & 1150769 & 363362 & SER356 & 2077 & 622032 & 255599 \\
\hline SEC38 & 1419 & 351652 & 264334 & GRR25 & 2344 & 1202773 & 2730081 & SCR61 & 2050 & 648672 & 615296 \\
\hline SEC36 & 1338 & 410593 & 225423 & GRR5 & 2067 & 1230909 & 1360404 & SCR63 & 1732 & 987481 & 25293 \\
\hline
\end{tabular}




\begin{tabular}{llllllllllll}
\hline SEC37 & 1301 & 417814 & 107090 & DAD14 & 998 & 2705531 & 145411 & SCR71 & 1588 & 1233190 & 49805 \\
SEC33 & 1204 & 580201 & 1086918 & GRR14 & 948 & 2854180 & 75555 & SCR50 & 1467 & 1415581 & 81853 \\
SIN526 & 1246 & 616948 & 415383 & DAD41 & 869 & 2888266 & 190487 & SER329 & 1401 & 1502168 & 72207 \\
SEC34 & 1013 & 770644 & 318664 & GRR12 & 915 & 2923003 & 106202 & SCR55 & 1328 & 1628340 & 85264 \\
SEC39 & 1093 & 861950 & 787719 & DAD37 & 890 & 2945471 & 101404 & SCR73 & 1243 & 1777709 & 42066 \\
SIN525 & 966 & 901756 & 473202 & DAD43 & 842 & 3101263 & 171831 & SER347 & 1219 & 1830676 & 142508 \\
SIN524 & 848 & 948366 & 376136 & DAD11 & 802 & 3270783 & 352718 & SER352 & 1171 & 1913818 & 411495 \\
SIN527 & 845 & 1194477 & 1053061 & GRR11 & 680 & 3361166 & 494934 & SEF28 & 1230 & 2095058 & 1640638 \\
CAL96 & 1405 & 403767 & 494010 & CAL96 & 1263 & 2081465 & 160283 & CAL96 & 1289 & 1739035 & 234438 \\
\hline
\end{tabular}

Note. ${ }^{\#} \mathrm{CS}=$ Cultivar superiority, $\mathrm{WE}=$ Wricke's ecovalence.

Considering location means, there was generally higher yields at Kachwekano than at Kawanda (Figure 6). Though many lines did not maintain stable yields in the two locations, some not only outperformed the local yield check (CAL96) but also maintained high yields at both locations. The most outstanding in this category include; DAB299, DAB291, DAB249, DAB231 (DAB Set2), DAB582 (DAB Set 2), SCN 14, SCN15, SCN16, SCN20 (Black beans), SEC40, SEC41 (Colored beans), DAD34, DAA7, GRR25 (Assorted), SCR66, SCR60, SCR46, SCR335, SER356 and SCR61 (Small-red beans) (Figure 6). Figure 7 shows the yield of 23 lines that maintained yields higher than the mean in all the four environments in comparison with the performance of CAL96. Some of the lines mentioned above appear in this category.
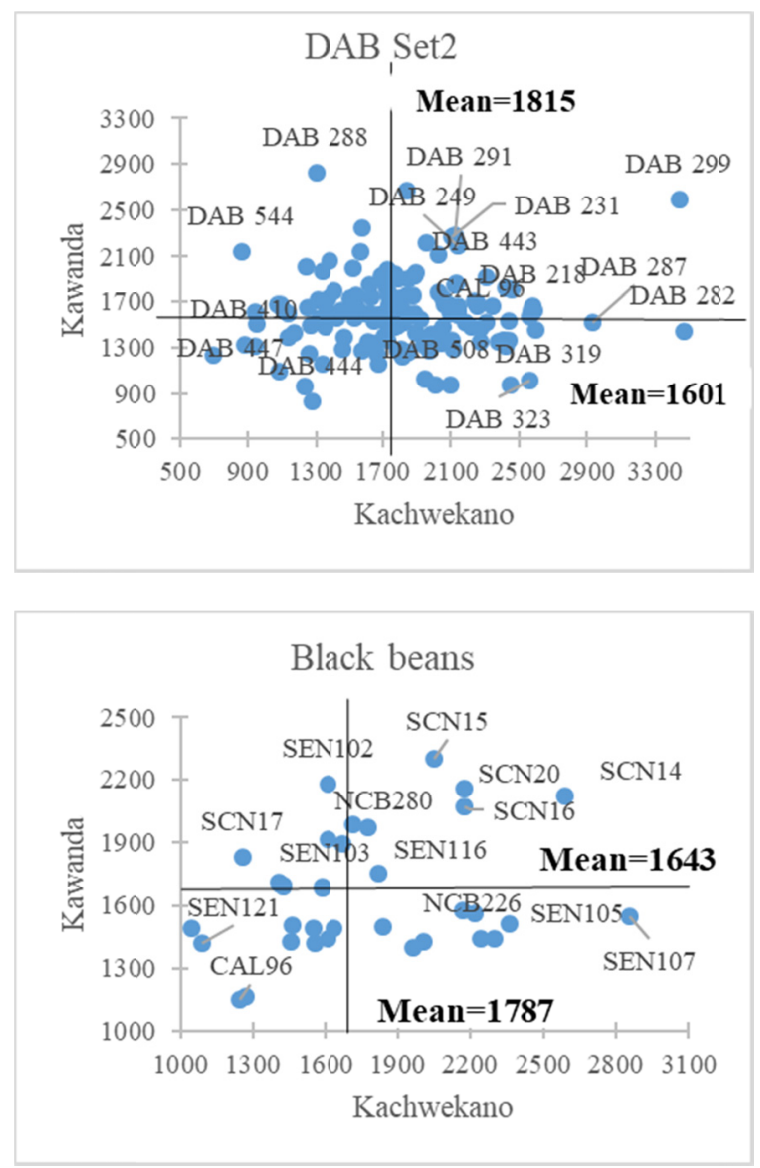
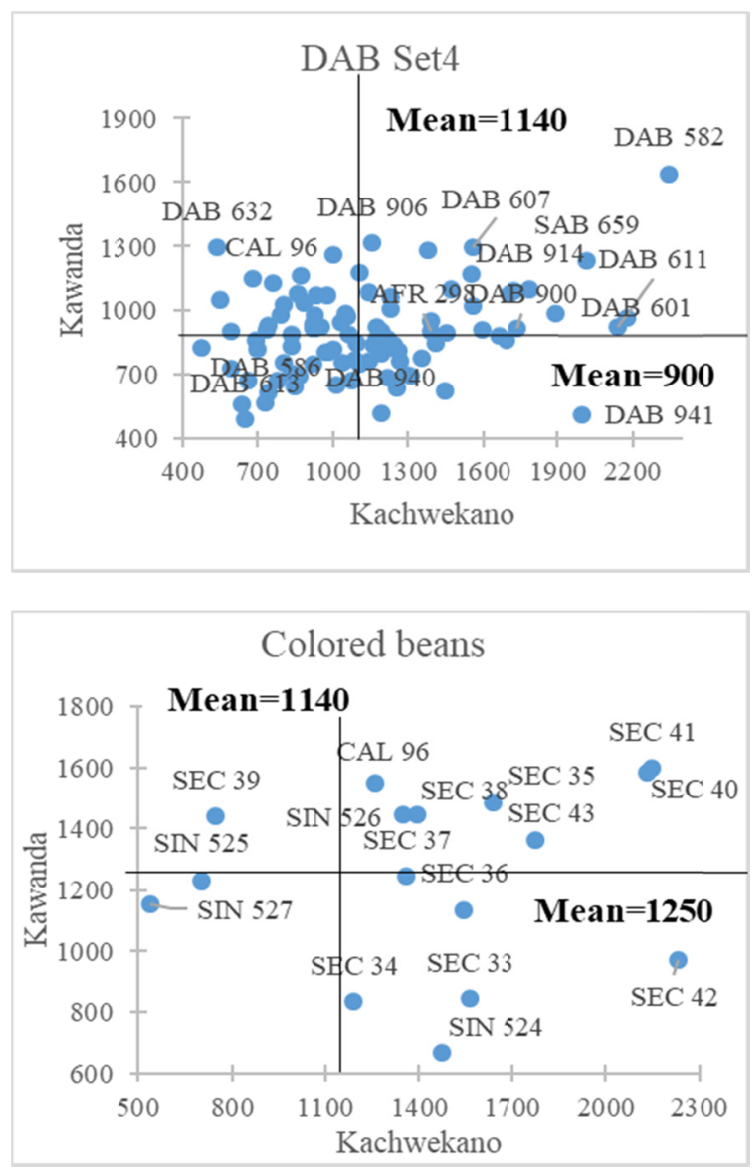

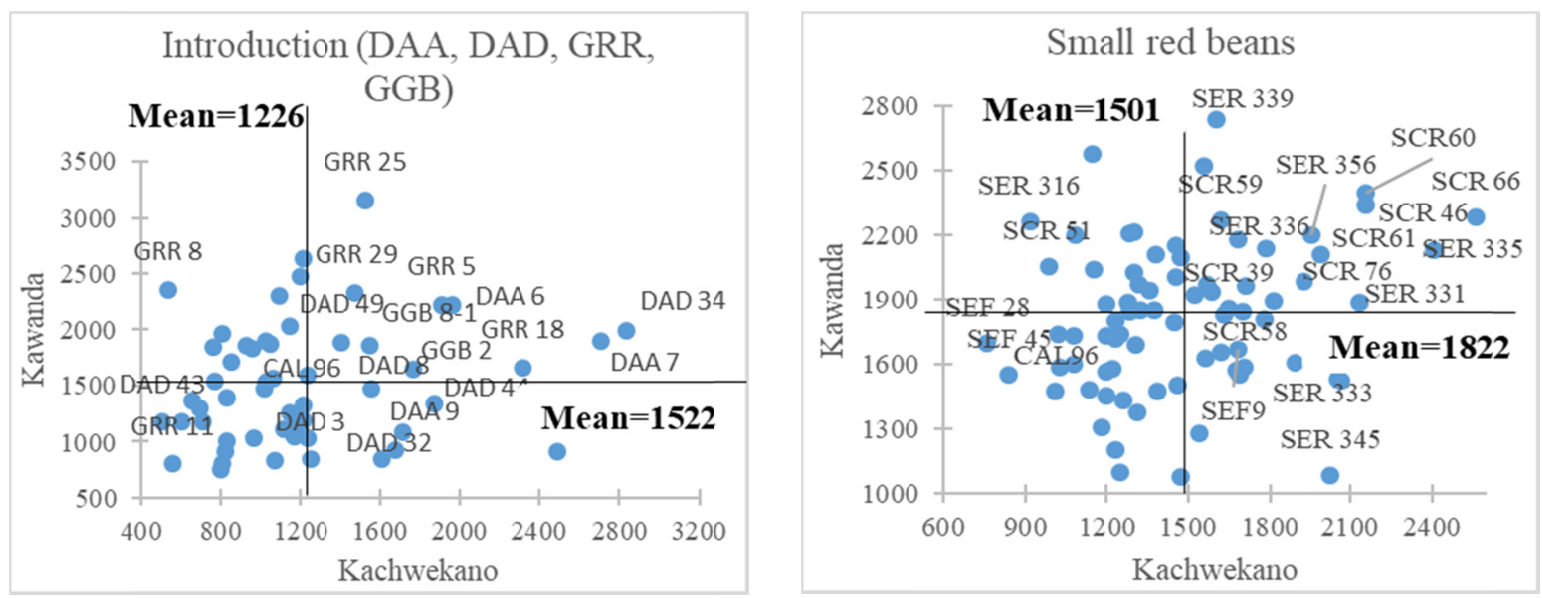

Figure 6. Comparison of yield performance of lines at Kawanda and Kachwekano

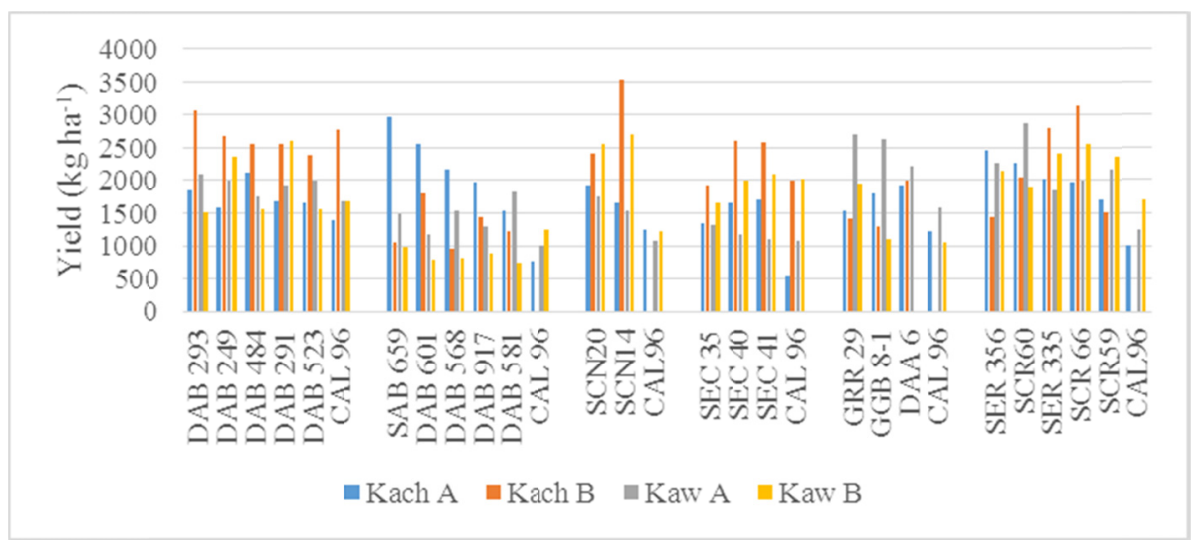

Figure 7. Yield performance of lines that maintained above average performance in all four the environments in comparison to the local yield check (CAL 96); $\mathrm{A}=$ first season, $\mathrm{B}=$ second season

\section{Discussion}

In consideration of the global and regional trends in climate change in the last few years, the development of drought-tolerant crop varieties is becoming more relevant in mitigating the effects of low water availability. Water stress is especially profound among small scale producers living in drought-prone areas, who cannot afford to apply irrigation. Releasing high-yielding and drought-tolerant bean cultivars would, thus, significantly contribute towards increased and stable yields in these areas. This study was aimed at identifying potential lines for use in breeding programs that are addressing drought challenges in the Pan African Bean Research Alliance (PABRA), especially in East and Central Africa. Trials were established in two locations during two rainy seasons. These environments were highly diverse because the same location generally grouped differently in different seasons. No precise grouping of environments suggests that more seasons and locations may need to consider to effectively select germplasm with wide adaptation. Seasons were expected to group as similar environments but this trend did not show in most traits possibly because of the effect of weather alterations. In DF and DPM, there was a clear difference in genotype (line) performance in the two locations. Kachwekano, located at 2200 masl registered higher values for these traits than Kawanda (1190 masl). It is evident that differences in altitude affected flowering and maturity time. George et al. (1990) confirmed that increasing elevation delays all phenological responses in soybean. Although other environmental factors like water stress and nutrient availability affect flowering and post flowering processes, temperature has been reported has the main influencer (Hodges \& French, 1985). In this study, delayed flowering and maturity of all the lines at 2200 $\mathrm{m}$ asl suggests that low temperature was the main contributing factor. In 2009, Alliprandini et al. found significant effect of altitude and latitude in maturity of soybean and noted that it is important to test lines in areas varying in these attributes for proper maturity grouping. Although few, some of the lines like DAB918 
maintained early maturity at both locations. Compared to the check and other released varieties, there were no exceptionally early or extremely late lines identified in the study.

Genotype by environment $(G \times E)$ interaction was very large in all the traits leading to high differences in genotype (line) performance in the trial environments. This explains the huge variations in performance ranks in the four environments. We used dynamic stability concept to describe $\mathrm{G} \times \mathrm{E}$ in the yield data. According to Norden, Gorbert, Knauft, and Martin, (1986) dynamic stability is expressed when a genotype's performance has no deviation from the general performance of all lines in the trial thus it is useful for quantitative traits such as yield. Lines that have the lowest Wricke's ecovalence were considered relatively stable because they have smaller deviations from the grand environment mean. Six of the 462 test lines; SCN20, SCN13, SEN114, SEC40 and SEC35 not only expressed yield stability but also superiority. However, only DAB901 which had an average across environment yield would be considered stable in this study if basing on Becker \& Leon, (1988). They only considered lines with zero Wricke's ecovalence as stable.

Many outstanding lines with a high variability for yield were identified but only those that were stable across seasons and or locations are more useful. The wide range observed between the local yield check (CAL96), and the highest yielding lines per group suggest that there are potential candidates for release or developing new breeding materials. Yield, a commercial trait to farmers, is a very important trait for pre-breeding planning because a high yielding variety is more likely to result in considerable yield gain if used for variety improvement Several lines in each of the groups outperformed CAL96 and $23(5.0 \%)$ of lines maintained above average yield in both locations and seasons. These lines are very promising considering that the average on-farm yields in East and Central Africa remains below $1000 \mathrm{~kg} \mathrm{ha}^{-1}$ (CIAT, 2008). Evaluation of un-adapted lines do not always result in high yield due to $\mathrm{G} \times \mathrm{E}$ interaction and other constraints that many be present in new environment. In this study, growth vigor for most lines was either good or excellent indicating high adaptability.

In the case of field diseases, majority of lines showed mild symptoms but those with severe infections were also observed. Since only natural disease infection was considered, the observed variations could be partly attributed to uneven disease pressure, but it is likely that several of these lines possess genetic resistance to the common prevailing diseases. Specifically, for common bacterial blight (CBB), majority of the lines in all the six groups except DAB Set4 had lower scores than CAL96, the susceptible check. Although varying levels for this disease was observed, most lines showed high to moderate resistance. It is important to confirm resistance of the superior lines to $\mathrm{CBB}$ in hot spots or to specific virulent isolates. Very few lines resistant/tolerant to CBB are in circulation in East and Central Africa (Wagara \& Kimani, 2007; Belarmino, 2015) and yet this is a major production constraint. Continuous screening for new sources of resistances for adoption and improvement of susceptible commercial varieties is required as a sustainable coping mechanism. Belarmino, (2015) and Alladassi, Nkalubo, Mukankusi, Kelly, and Urrea, (2016) identified resistant exotic lines that can be utilized for varietal improvement but majority of them are small seeded. The test lines used in this study are of several colors and sizes, and would meet several market preferences in addition to having drought tolerance in the background.

On the other hand, most lines seem susceptible to angular leaf spot (ALS) because they scored higher than CAL96, the susceptible check. ALS is one of the most devastating diseases of common bean with continuously emergency of new races, which break down disease resistance (Ddamulira et al., 2014a, 2014b). The low resistance levels we observed is not surprising since the lines were not specifically improved for ALS resistance. In fact, pyramiding of ALS resistance genes has been considered as a more effective way for providing durable resistance (Ddamulira et al., 2015) because of the diversity of the virulent races. Although confirmatory studies in hot spots are required, our study show the existence of multiple resistance in these breeding lines and this may result in wide adoption. In spite of symptoms being below economic damage levels, ascochyta blight, was observed as an emerging disease at Kachwekano, the highland location. With the alterations in weather, it may become one of the major constraints in a nearby future. Several studies have reported that the weather alterations being experienced have influenced the occurrence of certain biotic constraints (Garrett et al., 2006; Gautam et al., 2013; Paparu et al., 2014).

\section{Conclusion}

Yield consistency is an important attribute in varietal improvement but usually a low percentage of the best genotypes (lines) are stable. Promotion of these lines together with high performers in specific environments could significantly increase adoption. We identified several superior yielding lines in both categories with potential for release and varietal improvement. These germplasm will be distributed in the PABRA network for further testing and selection under drought conditions. Although this study is representing mid and high attitude areas, good vigor observed among lines suggests that they could easily adapt to a wider range of environments. 
Findings on field diseases indicate possible sources for angular leaf spot, common bacterial blight and rust resistance but there is need for confirmation under high disease pressure.

\section{Acknowledgements}

The Canadian International Development Agency (CIDA), Swiss Agency for Development and Cooperation (SDC) and United States Agency for International Development (USAID) are founding donors and continue to support the PABRA alliance. The Department for International Development (DFID) of UK, The Rockefeller Foundation, Bill and Melinda Gates Foundation, the Alliance for Green Revolution in Africa (AGRA), KirkHouse Trust, etc. also support components of the PABRA agenda. Chris Beadle from Commonwealth Scientific and Industrial Research Organization, Australia, reviewed the manuscript.

\section{References}

Acquaah, G. (2007). Principles of plant genetics and breeding (1st ed.). Blackwell Publishing Ltd: United Kingdom

Alliprandini, L. F., Abatti, C., Bertagnolli, P. F., Cavassim, J. E., Gabe, H. L., Kurek, A., ... Steckling, C. (2009). Understanding soybean maturity groups in Brazil: Environment, cultivar classification, and stability. Crop Sci, 49, 801-808. https://doi.org/10.2135/cropsci2008.07.0390

Amongi, W., Nkalubo, S. T., Ochwo-Ssemakula, M., Gibson, P. T., \& Edema, R. (2014). Development of intermittent drought stress tolerant common bean genotypes in Uganda. African Crop Science Journal, 22(4), 303-315.

Asfaw, A., \& Blair, M. W. (2014). Quantification of drought tolerance in Ethiopian common bean varieties. Agricultural Sciences, 5, 124-139. https://doi.org/10.4236/as.2014.52016

Badr, L. A. A. (2005). Inheritance and nature of drought tolerance in common bean (Phaseolus vulgaris L.) (Unpublished Master's thesis, Zagazig University, Benha Branch, Egypt).

Beebe, S. E., Rao, I. M., Cajiao, I., \& Grajales, M. (2008). Selection for drought resistance in common bean also improves yield in phosphorus limited and favourable environments. Crop Science, 48, 582-592. https://doi.org/10.2135/cropsci2007.07.0404

Beebe, S. E., Rao, I. M., Blair, W., \& Acosta-Gallegos, J. A. (2013). Phenotyping common beans for adaptation to drought. Font. Physiol, 4, 1-20. https://doi.org/10.3389/fphys.2013.00035

Becker, H. C., \& Leon, J. (1988). Stability analysis in plant breeding. Plant Breeding, 101(1), 1-23. https://doi.org/10.1111/j.1439-0523.1988.tb00261.x

Belarmino, D. (2015). Inheritance of resistance to common bacterial blight (Xanthomonas campestris pv. phaseoli) disease and yield of common bean (Unpublished Master's thesis, School of Agricultural Sciences, Makerere University, Kampala, Uganda).

Alladassi, B. M. E., Nkalubo, S. T., Mukankusi, M., Kelly, J., \& Urrea, C. (2016). Identification of common bacterial blight resistant sources for the bean-breeding program in Uganda. Presented at the Pan-African Grain Legume \& World Cowpea Conference in Livingstone, Zambia in March 2016. Retrieved from http:/www.pabra-africa.org/wp-content/uploads/dlm_uploads/2016/03/Identification-of-common-bacterialblight-resistant-sources-for-the-bean-breeding-program-in-Uganda-Alladassi-Makerere-NaCCRI.pdf

Broughton, W., Hernandez, G., Blair, M., Beebe, S., Gepts, P., \& Vanderleyden, J. (2003). Beans (Phaseolus spp.) model food legumes. Plant and Soil, 252, 55-61. https://doi.org/10.1023/A:1024146710611

Blair, M. W., Galeano, C. H., Tovar, E., Munoz Torres, M. C., Castrillon, A. V., Beebe, S. E., \& Rao, I. M. (2012). Development of a Mesoamerican intra-genepool genetic map for quantitative trait loci detection in a drought tolerant 3 susceptible common bean (Phaseolus vulgaris L.) cross. Mol Breeding, 29, 71-88. https://doi.org/10.1007/s11032-010-9527-9

Buruchara, R., Mukankusi, C., \& Ampofo, K. (2010). Bean disease and pest identification and management. Kampala, UG: International Center for Tropical Agriculture (CIAT); Pan-Africa Bean Research Alliance (PABRA). Handbooks for small-scale seed producers (No. 4). CIAT, Uganda.

Buruchara, R., Chirwa, R., Sperling, L., Mukankusi, C., Rubyogo, J. C., Muthoni, R., \& Abang, M. M. (2011). Development and delivery of bean varieties in Africa: The Pan-Africa Bean Research Alliance (PABRA) model. African Crop Science Journal, 19(4), 227-245. 
CIAT (Centro Internacional de Agricultura Tropical). (1987). In A. Van Schoonhoven, \& M. A. Pastor-Corrales (Eds.), Standard system for the evaluation of bean germplasm. Cali, Colombia.

CIAT (Centro Internacional de Agricultura Tropical). (2008). The impact of improved bush bean varieties in Uganda. Highlights-CIAT in Africa, 43. Kampala: CIAT.

Ddamulira, G., Mukankusi, C., Ochwo-Ssemakula, M., Edema, R., Sseruwagi, P., \& Gepts, P. (2014a). Distribution and variability of Pseudocercospora griseola in Uganda. Journal of Agricultural Science, 6(6), 16-29. https://doi.org/10.5539/jas.v6n6p16

Ddamulira, G., Mukankusi, C., Ochwo-Ssemakula, M., Edema, R., Sseruwagi, P., \& Gepts, P. (2014b). Identification of new sources of resistance to angular leaf spot among Uganda common bean landraces. Canadian Journal of Plant Breeding, 2(2), 55-65. https://doi.org/10.9734/AJEA/2015/17833

Ddamulira, G., Mukankusi, C., Ochwo-Ssemakula, M., Edema, R., Sseruwagi, P., \& Gepts, P. (2015). Gene pyramiding improved resistance to angular leaf spot in common bean. American Journal of Experimental Agriculture, 9(2), 1-12. https://doi.org/10.9734/AJEA/2015/17833

EAC (East African Community) Secretariat. (2011). East African community climate change master plan 2011-2013, Arusha, Tanzania.

Garrett, K. A., Dendy, S. P., Frank, E. E., Rouse, M. N., \& Travers, S. E. (2006). Climate change effects on plant disease: Genomes to ecosystems. Annu Rev Phytopathol, 44, 489-509. https://doi.org/10.1146/annurev. phyto.44.070505.143420

Gathu, E. W., Karuri, E. G., \& Njage, P. M. K. (2012). Physical characterization of new advanced drought tolerant common bean (Phaseolus vulgaris) lines for canning quality. American Journal of Food Technology 7, 22-28.

Gautam, H. R., Bhardwaj, M. L., \& Rohitashw, K. (2013). Climate change and its impact on plant diseases: Current Science, 105(12), 1685-1691.

GenStat. (2013). GenStat Release 16.2. GenStat Procedure Library, VSN International Ltd., Rothamsted, UK.

George, T., Bartholomew, D. P., \& Singleton, P. W. (1990). Effect of temperature and maturity group on phenology of field grown nodulating and nonnodulating soybean isolines. Biotronics, 19, 49-59.

Hodges, T., \& French, V. (1985). Soybean growth stages modeled from temperature, day length, and water availability. Agron. J., 77, 500-505. https://doi:10.2134/agronj1985.00021962007700030031x

Integrated Breeding Platform. (2013). Trait dictionary for Phaseolus Vulgaris L. Retrieved from https:/www.integratedbreeding.net/133/communities/genomics-crop-info/crop-information/beans

Kiwuka, C., Bukenya-Ziraba, R., Namaganda, M., \& Wasswa, M. J. (2012). Assessment of common bean cultivar diversity in selected communities of central Uganda. African Crop Science Journal, 20(4), 239-249.

Lin, C. S., \& Binns, M. R. (1988). A superiority performance measure of cultivar performance for cultivar $\mathrm{x}$ location data. Canadian Journal of Plant Science, 68, 193-198. https://doi.org/10.4141/cjps88-018

Mukankusi, C. (2008). Improving resistance to Fusarium root rot [Fusarium solani (Mart.) Sacc. f.sp Phaseoli (Burkholder) W.C. Synder \& H.N. Hans] in Common bean (Phaseolus vulgaris L.) (PhD thesis, University of Kwa Zulu-Natal, South Africa).

Munoz-Perea, G. C., Teran, H., Allen, R. G., Wright, J. L., Westermann, D. T., \& Singh, S. P. (2006). Selection for drought resistance in dry bean landraces and cultivars. Crop Science, 46, 2111-2120. https://doi.org/ 10.2135/cropsci2006.01.0029

NAPA (National Adaptation Programmes of Action). (2007). Climate change Uganda National Adaptation Programmes of Action. Kampala, Uganda.

Nkalubo, S., Melis, R., Laing, M. D., \& Opio, F. (2007). Yield loss associated with anthracnose disease on Ugandan market-class dry bean cultivars. African Crop Science Conference Proceedings, 8, 869-874.

Norden, A. J., Gorbert, D. W., Knauft, D. A., \& Martin, F. G. (1986). Genotype × environment interaction in peanut multiline population. Crop Sci, 26, 46-48. https://doi.org/10.2135/cropsci1986.0011183X0026 00010010x

Opio, F., Ugen, M. A., Kyamanywa, S., David, S., \& Mugisa-Mutetikka, M. (2001). Beans. In J. K. Mukiibi (Ed.), Agriculture in Uganda: Crops II. Fountain Publishers Kampala, Uganda. 
Paparu, P., Mukankusi, C., Nkalubo, S., Acur, A., Kato, F., Male, A., ... Ugen, M. A. (2014). Emerging disease problems: The case of southern blight of common beans. Presented at the second biennial National Agricultural Research Organization (NARO) scientists conference held from 3-7 November, 2014, at Speke Resort Hotel, Munyonyo, Kampala, Uganda. Retrieved from https://cgspace.cgiar.org/bitstream/ handle/10568/66110/Emerging_Disease_problems.pdf

Singh, S. P. (2007). Drought resistance in the race durango dry bean landraces and cultivars. Agronomy Journal, 99, 1219-1225. https://doi.org/10.2134/agronj2006.0301

Thomas, C. V. (1983). Genetic, morphological and physiological studies of drought and heat resistance in tepary bean (Phaseolus acutifolius A. Gray) and common bean (P. vulgaris L.). Dissertation Abstracts International, $B, 44(2), 410$.

UEPB (Uganda Export Promotion Board). (2005). Product Profile on Beans (No 12). Retrieved from

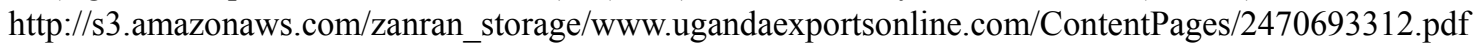

UNFCCC (United Nation Framework Convention on Climate Change). 2007. Climate change: Impacts, vulnerabilities, and adaptation in developing countries. Retrieved from https://www.preventionweb. net/files/2759_pub07impacts.pdf

Wagara, I. N., \& Kimani, P. M. (2007). Resistance of nutrient rich bean varieties to major biotic constraints in Kenya. African Crop Science Conference Proceedings, 18, 2087-2090.

White, J., \& Singh, S. P. (1991). Physiology of yield potential and stress tolerance. In A. Van Schoonhoven, \& O. Voysest (Eds.), Common bean: Research for crop improvement. CABI, Walingford, UK, and Centro Internacional de Agricultura Tropical, Cali, Colombia

Wricke, G. (1962). On a method of understanding the biological diversity in field research. Z. Pfl. Zucht, 47, 92-146.

Yan, W., Hunt, L. A., Sheng, Q., \& Szlavnics, Z. (2000). Cultivar Evaluation and Mega-Environment Investigation Based on the GGE Biplot. Crop Science, 40, 597-605. https://doi.org/10.2135/cropsci2000. $403597 x$

Yan, W., \& Kang, M. S. (2003). GGE Biplot Analysis: A Graphical Tool for Breeders, Geneticists and Agronomists. CRC Press, Boca Raton. https://doi.org/10.1201/9781420040371

Yan, W., Kang, M. S., Ma, B., Woods, S., \& Cornelius, P. L. (2007). GGE Biplot vs. AMMI Analysis of Genotype-by-Environment Data. Crop Science, 47, 643-653. https://doi.org/10.2135/cropsci2006.06.0374

\section{Copyrights}

Copyright for this article is retained by the author(s), with first publication rights granted to the journal.

This is an open-access article distributed under the terms and conditions of the Creative Commons Attribution license (http://creativecommons.org/licenses/by/4.0/). 